IMA Journal of Numerical Analysis (2006) 26, 96-130

doi:10.1093/imanum/dri023

Advance Access publication on August 9, 2005

\title{
Dual-primal FETI algorithms for edge finite-element approximations in 3D
}

\author{
ANDREA TOSELLI $\dagger$ \\ Seminar for Applied Mathematics, ETH Zürich, CH-8092 Zürich, Switzerland
}

[Received on 17 September 2004; revised on 18 March 2005]

\begin{abstract}
A family of dual-primal finite-element tearing and interconnecting methods for edge-element approximations in 3D is proposed and analysed. The key part of this work relies on the observation that for these finite-element spaces there is a strong coupling between degrees of freedom associated with subdomain edges and faces and a local change of basis is therefore necessary. The primal constraints are associated with subdomain edges. We propose three methods. They ensure a condition number that is independent of the number of substructures and possibly large jumps of one of the coefficients of the original problem, and only depends on the number of unknowns associated with a single substructure, as for the corresponding methods for continuous nodal elements. A polylogarithmic dependence is shown for two algorithms. Numerical results validating our theoretical bounds are given.
\end{abstract}

Keywords: edge elements; Maxwell's equations; finite elements; domain decomposition; FETI; preconditioners; heterogeneous coefficients.

\section{Introduction}

In this paper, we consider the boundary-value problem

$$
\begin{aligned}
L \mathbf{u}:=\operatorname{curl}(A \operatorname{curl} \mathbf{u})+B \mathbf{u} & =\mathbf{f} & & \text { in } \Omega, \\
\mathbf{n} \times(\mathbf{u} \times \mathbf{n}) & =0 & & \text { on } \partial \Omega,
\end{aligned}
$$

with $\Omega$ a bounded polyhedral domain in $\mathbb{R}^{3}$ and curl the 3D curl operator; see, e.g. Monk (2003). The domain $\Omega$ has unit diameter and $\mathbf{n}$ is its unit normal. The coefficient matrices $A$ and $B$ are symmetric, uniformly positive definite with entries $A_{i j}, B_{i j} \in L^{\infty}(\Omega), 1 \leqslant i, j \leqslant 3$.

The weak formulation of problem (1.1) requires the introduction of the Hilbert space $H(\mathbf{c u r l} ; \Omega)$, defined by

$$
H(\operatorname{curl} ; \Omega):=\left\{\mathbf{v} \in\left(L^{2}(\Omega)\right)^{3} \mid \operatorname{curl} \mathbf{v} \in L^{2}(\Omega)^{3}\right\} .
$$

The space $H(\mathbf{c u r l} ; \Omega)$ is equipped with the following inner product and graph norm,

$$
(\mathbf{u}, \mathbf{v})_{\text {curl }}:=\int_{\Omega} \mathbf{u} \cdot \mathbf{v} \mathrm{d} x+\int_{\Omega} \operatorname{curl} \mathbf{u} \cdot \operatorname{curl} \mathbf{v} \mathrm{d} x, \quad\|\mathbf{u}\|_{\text {curl }}^{2}:=(\mathbf{u}, \mathbf{u})_{\text {curl }} .
$$

The tangential component $\mathbf{n} \times(\mathbf{u} \times \mathbf{n})=\mathbf{u}-(\mathbf{u} \cdot \mathbf{n}) \mathbf{n}$, of a vector $\mathbf{u} \in H(\mathbf{c u r l} ; \Omega)$ on the boundary $\partial \Omega$, belongs to the space $H^{-\frac{1}{2}}(\partial \Omega)^{3}$; see Brezzi \& Fortin (1991) and Monk (2003). We note that the vectors $\mathbf{n} \times(\mathbf{u} \times \mathbf{n})$ and $\mathbf{u} \times \mathbf{n}$ are perpendicular, have the same length and are both perpendicular to n. Boundary conditions can therefore be equivalently expressed in terms of either one. The trace space can be further characterized and this will be done in Section 7. The subspace of vectors in $H($ curl; $\Omega$ ) with vanishing tangential component on $\partial \Omega$ is denoted by $H_{0}($ curl; $\Omega)$.

\footnotetext{
†Email: toselli@sam.math.ethz.ch
} 
For any $\mathcal{D} \subset \Omega$, we define the bilinear form

$$
a_{\mathcal{D}}(\mathbf{u}, \mathbf{v}):=\int_{\mathcal{D}}(A \operatorname{curl} \mathbf{u} \cdot \operatorname{curl} \mathbf{v}+B \mathbf{u} \cdot \mathbf{v}) \mathrm{d} x, \quad \mathbf{u}, \mathbf{v} \in H(\operatorname{curl} ; \Omega) .
$$

The variational formulation of (1.1) is as follows.

Find $\mathbf{u} \in H_{0}(\mathbf{c u r l} ; \Omega)$ such that

$$
a_{\Omega}(\mathbf{u}, \mathbf{v})=\int_{\Omega} \mathbf{f} \cdot \mathbf{v} \mathrm{d} x, \quad \mathbf{v} \in H_{0}(\operatorname{curl} ; \Omega) .
$$

Neumann and/or inhomogeneous conditions can also be considered and the generalization of our algorithms to these cases is straightforward.

The purpose of this work is to construct and analyse a dual-primal finite-element tearing and interconnecting (FETI-DP) preconditioner for 3D $h$-version finite-element approximations of problem (1.3). Neumann-Neumann (NN) and FETI algorithms are particular domain decomposition (DD) methods of iterative substructuring type: they rely on a non-overlapping partition into subdomains. They are among the most popular and heavily tested DD methods and are now employed for the solution of huge problems on parallel architectures; see, e.g Mandel \& Brezina (1996), Farhat \& Roux (1994), Farhat et al. (2001), Traore et al. (2002) and Bhardwaj et al. (2002). The rate of convergence is often independent of possibly large jumps in the coefficients.

The motivation of this work lies in the fact that devising iterative substructuring methods (and in particular NN or FETI preconditioners) for 3D edge-element approximations, that are robust with respect to the number of unknowns, the number of subdomains and large jumps of the coefficients, has turned out to be a particularly hard problem in recent years. Some methods are available for 2D approximations. In Toselli et al. (2001), a DD preconditioner was proposed, which is based on a standard coarse space and local spaces associated with the subdomain edges. NN preconditioners with standard coarse spaces were studied in Toselli (2000a). One-level FETI methods were developed in Toselli \& Klawonn (2001) and Rapetti \& Toselli (2001), thanks with the introduction of suitable local functions which are the analogues of constants and rigid body modes for the Laplace equation and linear elasticity, respectively. These functions were then employed to construct a balancing NN method in Toselli (2000b). FETI-DP algorithms were proposed in Toselli \& Vasseur (2004). Standard coarse spaces, however, are not in general suitable for quasi-optimal preconditioners in 3D and the search for suitable local functions in $3 \mathrm{D}$ for balancing NN and one-level FETI methods has produced no results so far.

A first important attempt to solve this problem was made in Hu \& Zou (2003), where a wire basket algorithm was proposed and studied in 3D. Local components are associated with the faces of the partition and two wire basket coarse spaces are then considered. The underlying idea is to employ two coarse spaces that should reduce two error components associated with a discrete Helmholtz decomposition. The corresponding preconditioned operator is shown to be scalable and its condition number to grow polylogarithmically. Independence of coefficient jumps is not guaranteed due to the overlap between the coarse and local spaces. No numerical result is provided. In a subsequent paper by Hu \& Zou (2004), generalizations to a saddle-point problem and to cases with large coefficient jumps were proposed, together with a different coarse space/solve. We also refer to Butrylo et al. (2004), which gives a fine survey of algebraic solvers currently employed in large-scale computational electromagnetics. We note in particular that some FETI algorithms are sometimes employed in practice (see (4.4)) without preconditioning.

In this work, we first show that the difficulty of iterative substructuring for edge-element approximations mainly lies in the strong coupling between degrees of freedom associated with subdomain edges 
and faces and that no efficient and robust iterative substructuring strategy is therefore possible unless a change of basis is performed. The situation here is the same as for general $p$ - and $h p$-version continuous finite-element approximations for diffusive problems, for which strong coupling exists between edge and face components, unless very special bases are employed; see, e.g. Mandel (1990). The surprising feature is that strong coupling appears here even for the simple $h$-version, something that has no analogue with nodal finite elements.

We next propose a change of basis, which is local and is only associated with the tangential degrees of freedom on the subdomain edges. This change of basis is much simpler than those necessary for $p$-versions, produces very sparse matrices and does not rely on the fact that the subdomains have a simple shape.

Once a change of basis is performed we are able to devise robust FETI-DP algorithms, by selecting suitable primal constraints quite straightforwardly. We propose three algorithms: one with a minimal coarse space that does not exhibit good convergence properties for large problems, one with an improved, low-dimensional coarse space for which good convergence is observed and a third one with a very large coarse space, which has the advantage of not requiring a change of basis and can therefore be potentially suitable for some future extensions.

An analysis of the resulting condition numbers is performed: it relies on some generalizations of certain discrete Sobolev-type inequalities for the more complicated trace spaces associated with the subdomains $\Omega_{i}$, conforming in $\mathbf{H}_{\perp}^{-1 / 2}\left(\operatorname{curl}_{S} ; \Omega_{i}\right)$. The bounds are not optimal, but we present them here in view of the novelty of these methods and of these theoretical tools.

This paper is organized as follows: In Section 2, we introduce our discrete problems, the subdomain partition and local and global finite-element spaces. Tangential vector finite-element spaces are described in Section 3. We introduce FETI-DP algorithms and an abstract framework for their analysis in Section 4. The strong coupling between edge and face degrees of freedom is shown in Section 5. Our FETI-DP algorithms, which rely on a change of basis and suitable sets of primal constraints, are introduced in Section 6. Section 7 is devoted to the technical tools necessary for the analysis: they consist of trace and inverse inequalities for discrete tangential vectors, decompositions associated with the face boundaries and the face interiors and relations between these decompositions for neighbouring substructures. The proofs of two of the main results are given in the appendices. Condition number bounds are given in Section 8 and Section 9 is devoted to some numerical tests.

\section{Partitions and discrete spaces}

We discretize this problem using edge elements, also known as Nédélec elements; see Nédélec (1980). These are vector finite elements that only ensure the continuity of the tangential component across the elements, as is physically required for the electric and magnetic fields, solutions of Maxwell's equations. We refer to Monk (2003) for a fine presentation of approximations of electromagnetic problems, the Sobolev space $H($ curl; $\Omega$ ) and edge elements.

We introduce a shape-regular triangulation $\mathcal{T}=\mathcal{T}_{h}$ of the domain $\Omega$, made of affinely mapped cubes. In particular, if $\widehat{Q}=(-1,1)^{3}$ is a reference cube, for each element $K \in \mathcal{T}$, there exists an affine mapping $F_{K}: \widehat{Q} \rightarrow K$, such that $K$ is the image of $\widehat{Q}$. Here, we only consider meshes built on affinely mapped cubes for simplicity but our results are equally valid for approximations on tetrahedral meshes.

Let $\mathcal{E}_{h}$ be the set of edges of $\mathcal{T}$. For every edge $e \in \mathcal{E}_{h}$, we fix a direction, given by a unit vector $\mathbf{t}_{e}$, tangent to $e$. The length of the edge $e$ is denoted by $|e|$ and, in the following, we will always denote the measure of a region $\mathcal{D}$ by $|\mathcal{D}|$. 
We next consider a non-overlapping partition of the domain $\Omega$ into subdomains (substructures),

$$
\left\{\Omega_{i} \mid 1 \leqslant i \leqslant N, \bigcup_{i=1}^{N} \overline{\Omega_{i}}=\bar{\Omega}\right\},
$$

such that each $\Omega_{i}$ is connected. The substructures $\Omega_{i}$ are unions of elements in $\mathcal{T}$. We denote the diameter and the local mesh size of $\Omega_{i}$ by $H_{i}$ and $h_{i}$, respectively. We define $H$ as the maximum of the diameters of the subdomains:

$$
H:=\max _{1 \leqslant i \leqslant N}\left\{H_{i}\right\}
$$

We assume that the coefficients $A$ and $B$ are constant in each substructure $\Omega_{i}$ and denote them by $A_{i}$ and $B_{i}$, respectively. Since jumps of both coefficients will play a role in the rate of convergence of our algorithms, we only consider jumps in one of them:

$$
\begin{array}{ll}
\text { Case 1: } & \left\{\begin{array}{l}
A=\operatorname{diag}\{a, a, a\}, \\
0<\beta_{i}|\mathbf{x}|^{2} \leqslant \mathbf{x}^{t} B_{i} \mathbf{x} \leqslant \gamma_{i}|\mathbf{x}|^{2}, \quad \mathbf{x} \in \mathbb{R}^{3},
\end{array}\right. \\
\text { Case 2: } & \left\{\begin{array}{l}
0<\beta_{i}|\mathbf{x}|^{2} \leqslant \mathbf{x}^{t} A_{i} \mathbf{x} \leqslant \gamma_{i}|\mathbf{x}|^{2}, \quad \mathbf{x} \in \mathbb{R}^{3}, \\
B=\operatorname{diag}\{b, b, b\},
\end{array}\right.
\end{array}
$$

for $i=1, \ldots, N$, where $|\cdot|$ denotes the standard Euclidean norm. Scaling matrices for our FETI algorithms will be constructed with the values $\left\{\gamma_{i}\right\}$.

We always assume that the substructures are images of a reference square under sufficiently regular maps, which effectively means that their aspect ratios remain uniformly bounded. In addition, we assume that the ratio of the diameters of two adjacent subregions is bounded away from zero and infinity. Further assumptions, necessary for the analysis but not for the definition of the algorithms, are made at the beginning of Section 7 .

We next define the local spaces

$$
\mathrm{H}_{\star}\left(\operatorname{curl} ; \Omega_{i}\right):=\left\{\mathbf{u}_{i} \in \mathrm{H}\left(\operatorname{curl} ; \Omega_{i}\right) \mid \mathbf{n} \times\left(\mathbf{u}_{i} \times \mathbf{n}\right)=0 \text { on } \partial \Omega \bigcap \partial \Omega_{i}\right\}
$$

and the following polynomial spaces on the reference square,

$$
N D(\widehat{Q})=\mathbb{Q}_{0,1,1}(\widehat{Q}) \otimes \mathbb{Q}_{1,0,1}(\widehat{Q}) \otimes \mathbb{Q}_{1,1,0}(\widehat{Q}),
$$

with $\mathbb{Q}_{k_{1}, k_{2}, k_{3}}(\widehat{Q})$ the space of polynomials of degree $k_{i}$ in the $i$-th variable. We note that the tangential component of a vector in $N D(K)$ over a face of $\widehat{Q}$ perpendicular to e.g. the $x$ axis is a vector function contained in $\mathbb{Q}_{0,1} \times \mathbb{Q}_{1,0}$.

On an affinely mapped element $K \in \mathcal{T}$, we take

$$
N D(K)=\left\{\mathbf{u}=J_{F_{K}}^{-T} \widehat{\mathbf{u}} \mid \widehat{\mathbf{u}} \in N D(\widehat{Q})\right\},
$$

with $J_{F_{K}}$ the Jacobian of the transformation $F_{K}$. The tangential component of a vector in $N D(K)$ can also be characterized in this case.

On each subdomain $\Omega_{i}$, the lowest-order Nédélec finite-element spaces are defined as

$$
X_{i}=N D^{h}\left(\Omega_{i}\right):=\left\{\mathbf{u} \in \mathrm{H}_{\star}\left(\operatorname{curl} ; \Omega_{i}\right) \mid \mathbf{u}_{\left.\right|_{K}} \in N D(K), K \in \mathcal{T}_{h}, K \subset \Omega_{i}\right\} .
$$


Higher polynomial degrees can also be considered and our results and bounds will remain valid with constants that depend on the polynomial degree. See, e.g. Monk (2003) for more details. Functions in $X_{i}$ have a constant tangential component along the fine edges in $\mathcal{E}_{h}$. The degrees of freedom are normally chosen as the constant values of the tangential component on the fine edges in $\mathcal{E}_{h}$ : for $\mathbf{u} \in X_{i}$,

$$
\lambda_{e}(\mathbf{u}):=\mathbf{u} \cdot \mathbf{t}_{\left.e\right|_{e}}=|e|^{-1} \int_{e} \mathbf{u} \cdot \mathbf{t}_{e} \mathrm{~d} s, \quad e \in \mathcal{E}_{h}, e \subset \bar{\Omega}_{i}
$$

These edge averages also define local interpolation operators. The degrees of freedom (2.3) can be naturally partitioned into three classes according to where the corresponding edge lies: face, edge and interior.

We next consider the product space

$$
X=X(\Omega):=\prod_{i=1}^{N} X_{i} \subset \prod_{i=1}^{N} \mathrm{H}_{\star}\left(\operatorname{curl} ; \Omega_{i}\right),
$$

which consists of vectors that have in general a discontinuous tangential component across the subdomain boundaries. The discrete solution is sought in the conforming space

$$
\widehat{X}:=X \bigcap \mathrm{H}_{0}(\operatorname{curl} ; \Omega)
$$

of vectors with a continuous tangential component across the subdomain faces and edges.

Finally, we will also employ the standard finite-element spaces of scalar, continuous, piecewise trilinear functions on the subdomains $V^{h}\left(\Omega_{i}\right) \subset H^{1}\left(\Omega_{i}\right)$. We note that

$$
\nabla V^{h}\left(\Omega_{i}\right) \subset N D^{h}\left(\Omega_{i}\right) .
$$

\section{Interface functions}

We define the boundaries $\Gamma_{i}=\partial \Omega_{i} \backslash \partial \Omega$ and the interface $\Gamma$ as their union. We remark that $\Gamma$ is the union of the interior subdomain faces, regarded as open sets, which are shared by two subregions, and interior subdomain edges and vertices, which are shared by more than two subregions. In the following, we tacitly assume that points on $\partial \Omega$ are excluded from the geometrical objects that we consider, or in other words, we will only deal with geometrical objects (faces, edges, vertices, ...) that belong to $\Gamma$. We denote the faces of $\Omega_{i}$ by $F_{i j}$ and its edges by $E_{i j}$ and also use faces and edges with one or no subscript. We will always assume that a face $F_{i j}$ does not coincide with a connected component of $\partial \Omega_{i}$; this implies that the boundary $\partial F_{i j}$ is not empty.

For a face $F$ and an edge $E$ of a substructure $\Omega_{i}$, we introduce unit vectors tangential to $\partial F$ and $E$, denoted $\mathbf{t}_{\partial F}$ and $\mathbf{t}_{E}$, respectively. The sets of fine edges (and the corresponding degrees of freedom) on $\Gamma_{i}$ and $\Gamma$ are denoted by $\Gamma_{i, h}$ and $\Gamma_{h}$, respectively. We note that there are no degrees of freedom associated with the subdomain vertices and that subdomain faces and edges that lie on $\partial \Omega$ do not belong to the interface.

REMARK 3.1 When Neumann boundary conditions are imposed on $\partial \Omega$, edges (but not faces) lying on $\partial \Omega$ are part of the interface $\Gamma$ and also need to be employed in the definition of our algorithms.

We now introduce some trace spaces consisting of tangential components on the boundaries of the substructures. A tangential vector $\mathbf{w}$, defined on $\partial \Omega_{i} \backslash \partial \Omega$, belongs to $W_{i}$ if and only if there exists 
$\mathbf{u} \in X_{i}$ such that, on the closure of each face $F \subset \Gamma_{i}$,

$$
\mathbf{w}=\mathbf{n} \times(\mathbf{u} \times \mathbf{n}) .
$$

We note that a function $\mathbf{w} \in W_{i}$ belongs to the lowest-order, 2D edge-element space on each face $F$ and that the tangential component of $\mathbf{w}$ along an edge shared by two faces must be the same when calculated on either one of the faces.

Similarly, given a face $F$ of a substructure $\Omega_{i}$, we consider the tangential component along $\partial F$

$$
u=\mathbf{u} \cdot \mathbf{t}_{\partial F}, \quad \text { on } \partial F .
$$

The function $u$ is piecewise constant. A similar definition holds for the tangential component along an edge $E \subset \partial F$.

We will use the following convention: given a vector $\mathbf{u}$ defined in $\Omega_{i}$, we denote its tangential component on $\partial \Omega_{i}$ by the same bold letter $\mathbf{u}$. Its tangential component along an edge or the boundary of a face is denoted by $u$.

We will employ the product space of functions defined on $\Gamma, W:=\prod_{i} W_{i}$, and its continuous subspace $\widehat{W}$ consisting of tangential traces of vectors in $\widehat{X}$.

Vectors in the spaces $W_{i}$ and $W$ are uniquely defined by the degrees of freedom in $\Gamma_{i, h}$ and $\Gamma_{h}$, respectively. For each fine edge $e \in \Gamma_{h}$, let $\mathcal{N}_{e}$ be the set of indices of the subdomains that have $e$ on their boundary. Throughout this paper, we will use the same notation for a vector in $W_{i}$ or $X_{i}$ and the corresponding column vector of degrees of freedom. Similarly for the corresponding spaces and for global vectors in $X$ and $W$.

We recall that, if $\mathbf{u}=\nabla \phi \in H\left(\operatorname{curl} ; \Omega_{i}\right)$, for $\phi \in H^{1}\left(\Omega_{i}\right)$, and $\mathbf{v} \in H\left(\operatorname{curl} ; \Omega_{i}\right)$, then on ace $F$, we have (see Buffa \& Ciarlet (2001b, Section 2)),

$$
\begin{aligned}
& \mathbf{u}=\mathbf{n} \times(\nabla \phi \times \mathbf{n})=\nabla_{S} \phi=\nabla_{S}\left(\left.\phi\right|_{F}\right), \\
& \mathbf{c u r l} \mathbf{v} \cdot \mathbf{n}=\operatorname{curl}_{S} \mathbf{v}=\operatorname{curl}_{S}(\mathbf{n} \times(\mathbf{v} \times \mathbf{n})),
\end{aligned}
$$

with $\nabla_{S}$ and $\operatorname{curl}_{S}$ as the surface gradient and curl on $F$, respectively. For the whole of $\partial \Omega_{i}$, tangential gradients and curls are taken face by face.

Finally, for $i=1, \ldots, N$, we define the extensions into the interior of the $\Omega_{i}$

$$
\mathcal{H}_{i}: W_{i} \longrightarrow X_{i}
$$

that are discrete harmonic with respect to the bilinear forms $a_{\Omega_{i}}(\cdot, \cdot)$. We recall that $\mathbf{u}^{(i)}=\mathcal{H}_{i} \mathbf{w}^{(i)}$ minimizes the energy $a_{\Omega_{i}}\left(\mathbf{u}^{(i)}, \mathbf{u}^{(i)}\right)$ among all the vectors of $X_{i}$ with tangential component equal to $\mathbf{w}^{(i)}$ on $\Gamma_{i}$. We will refer to $\mathcal{H}_{i}$ as the Maxwell discrete harmonic extension.

\section{FETI-DP methods}

In this section, we introduce a first FETI-DP method for the solution of the linear system arising from the edge-element discretization of problem (1.3). Throughout the paper, given two column vectors $\mathbf{u}$ and $\mathbf{w}$ of degrees of freedom, we denote their scalar product in $l^{2}$ by $\langle\mathbf{u}, \mathbf{w}\rangle:=\mathbf{u}^{T} \mathbf{w}$. We recall that FETI-DP methods were originally introduced in Farhat et al. (2001). The first theoretical result was given in Mandel \& Tezaur (2001) for 2D problems and then later in Klawonn et al. (2002) for 3D. Extensive work and analysis has been performed for linear elasticity problems in Farhat et al. (2000) 
and Klawonn \& Widlund (2004). See also Toselli \& Widlund (2004, Chapters 6 and 8). Algorithms for 2D edge-element approximations have been proposed in Toselli \& Vasseur (2004).

We first assemble the local stiffness matrices, relative to the bilinear forms $a_{\Omega_{i}}(\cdot, \cdot)$, and the local load vectors. The degrees of freedom that belong only to one substructure can be eliminated in parallel by block Gaussian elimination. We note that these are degrees of freedom associated with edges $e$ in the interior of the substructures. We are then left with the degrees of freedom involving the tangential component along the substructure boundaries. Let $\mathbf{f}^{(i)}$ be the resulting right-hand sides and $S^{(i)}$ the Schur complement matrices

$$
S^{(i)}: W_{i} \longrightarrow W_{i},
$$

relative to the tangential degrees of freedom on $\Gamma_{i}$.

We recall that the local Schur complements satisfy the following property

$$
\left|\mathbf{u}^{(i)}\right|_{S^{(i)}}^{2}:=\left\langle\mathbf{u}^{(i)}, S^{(i)} \mathbf{u}^{(i)}\right\rangle=a_{\Omega_{i}}\left(\mathcal{H}_{i} \mathbf{u}^{(i)}, \mathcal{H}_{i} \mathbf{u}^{(i)}\right) ;
$$

see, e.g. Smith et al. (1996) and Toselli (2000a). Since the local bilinear forms are positive definite, so are the local Schur complements $S^{(i)}$. We write

$$
\mathbf{u}:=\left[\begin{array}{c}
\mathbf{u}^{(1)} \\
\vdots \\
\mathbf{u}^{(N)}
\end{array}\right] \in W, \quad S:=\operatorname{diag}\left\{S^{(1)}, \ldots, S^{(N)}\right\}, \quad \mathbf{f}:=\left[\begin{array}{c}
\mathbf{f}^{(1)} \\
\vdots \\
\mathbf{f}^{(N)}
\end{array}\right]
$$

The solution $\mathbf{u} \in W$ to the discrete problem can then be found by minimizing the energy

$$
\frac{1}{2}\langle\mathbf{u}, S \mathbf{u}\rangle-\langle\mathbf{f}, \mathbf{u}\rangle
$$

subject to the constraint that $\mathbf{u}$ is continuous, i.e. it belongs to $\widehat{W}$.

For FETI-DP methods, we work in a subspace $\widetilde{W} \subset W$ of functions satisfying a certain number of continuity constraints. We have

$$
\widetilde{W}=\widehat{W}_{\Pi} \oplus \widetilde{W}_{\Delta} .
$$

Here, the primal space $\widehat{W}_{\Pi} \subset \widehat{W}$ consists of continuous tangential vectors determined by degrees of freedom (primal variables) associated with the substructures. Choices for primal constraints are given in Section 6.2.

The dual space $\widetilde{W}_{\Delta}$ is the product space of spaces associated with the substructures

$$
\widetilde{W}_{\Delta}:=\prod_{i=1}^{N} \widetilde{W}_{\Delta, i}
$$

of functions for which the functionals given by the primal variables vanish.

The primal degrees of freedom can then be eliminated together with the internal ones, at the expense of solving one coarse problem. We are then left with a problem involving interface functions with vanishing primal degrees of freedom, and consequently, posed in the dual space $\widetilde{W}_{\Delta}$. Let $\widetilde{S}: \widetilde{W}_{\Delta} \rightarrow \widetilde{W}_{\Delta}$ be the corresponding Schur complement and $\widetilde{\mathbf{f}}_{\Delta}$ the corresponding load vector. We then look for $\mathbf{u}_{\Delta} \in$ $\widetilde{W}_{\Delta}$, such that

$$
\frac{1}{2}\left\langle\mathbf{u}_{\Delta}, \widetilde{S} \mathbf{u}_{\Delta}\right\rangle-\left\langle\widetilde{\mathbf{f}}_{\Delta}, \mathbf{u}_{\Delta}\right\rangle \longrightarrow \min
$$


subject to the constraint that $\mathbf{u}_{\Delta}$ is continuous. The continuity constraint is expressed by the equation

$$
B_{\Delta} \mathbf{u}_{\Delta}=0
$$

where $B_{\Delta}$ is constructed from $\{0,1,-1\}$ and evaluates the difference between all the corresponding tangential degrees of freedom on $\Gamma$. We employ the same matrix as in our previous paper (Toselli \& Klawonn, 2001) and then enforce redundant conditions associated with the substructure edges. The matrix $B_{\Delta}$ has the following block structure:

$$
B_{\Delta}=\left[\begin{array}{llll}
B_{\Delta}^{(1)} & B_{\Delta}^{(2)} & \cdots & B_{\Delta}^{(N)}
\end{array}\right]
$$

where each block corresponds to a substructure.

We obtain the saddle-point problem

$$
\begin{aligned}
\widetilde{S} \mathbf{u}_{\Delta}+B_{\Delta}^{\top} \lambda & =\widetilde{\mathbf{f}}_{\Delta}, \\
B_{\Delta} \mathbf{u}_{\Delta} & =0,
\end{aligned}
$$

with $\mathbf{u}_{\Delta} \in \widetilde{W}_{\Delta}$ and $\lambda \in V:=\operatorname{Range}\left(B_{\Delta}\right)$.

We note that $\widetilde{S}$ can be obtained from the restriction of $S$ to the space $\widetilde{W}$, by eliminating the primal degrees of freedom. We have therefore the minimization property

$$
\left\langle\mathbf{u}_{\Delta}, \widetilde{S} \mathbf{u}_{\Delta}\right\rangle=\min \langle\mathbf{u}, S \mathbf{u}\rangle
$$

where the minimum is taken over all the functions $\mathbf{u}=\mathbf{u}_{\Delta}+\mathbf{w}_{\Pi}, \mathbf{w}_{\Pi} \in \widehat{W}_{\Pi}$. This property ensures that $\widetilde{S}$ is also positive definite.

Since the Schur complement $\widetilde{S}$ is invertible, an equation for $\lambda$ can easily be found:

$$
F \lambda=d,
$$

with

$$
F:=B_{\Delta} \widetilde{S}^{-1} B_{\Delta}^{\top}, \quad d:=B_{\Delta} \widetilde{S}^{-1} \widetilde{f}_{\Delta}
$$

Once $\lambda$ is found, the primal variables are given by

$$
\mathbf{u}_{\Delta}=\widetilde{S}^{-1}\left(\widetilde{\mathbf{f}}_{\Delta}-B_{\Delta}^{\top} \lambda\right) \in \widetilde{W}
$$

In order to define a preconditioner for (4.4), we need to define scaling matrices and functions defined on the subdomain boundaries. In contrast with our previous work, (Toselli \& Klawonn, 2001; Toselli \& Vasseur, 2004), they are constructed with the coefficient that has jumps across the subdomains. For either case considered in (2.1), we define, on each substructure, $\delta_{i}^{\dagger} \in W_{i}$, such that on each fine edge $e \in \Gamma_{i, h}$,

$$
\delta_{\left.i\right|_{e}}^{\dagger}=\gamma_{i}^{\chi} / \sum_{j \in \mathcal{N}_{e}} \gamma_{j}^{\chi},
$$

for an arbitrary but fixed $\chi \in[1 / 2,+\infty)$; see (2.1). By direct calculation, we find

$$
\gamma_{i} \delta_{j}^{\dagger^{2}} \leqslant \min \left(\gamma_{i}, \gamma_{j}\right)
$$


For each substructure $\Omega_{i}$, we next introduce a diagonal matrix $D_{\Delta}^{(i)}: V \rightarrow V$. The diagonal entry corresponding to the Lagrange multiplier that enforces the equality of the degree of freedom associated with a fine edge $e$ between $\Omega_{i}$ and a second substructure $\Omega_{j}$ is set equal to the value of $\delta_{j}^{\dagger}$ along $e$. The remaining values are zero. We next define the scaled matrix

$$
B_{D, \Delta}=\left[\begin{array}{llll}
D_{\Delta}^{(1)} B_{\Delta}^{(1)} & D_{\Delta}^{(2)} B_{\Delta}^{(2)} & \cdots & D_{\Delta}^{(N)} B_{\Delta}^{(N)}
\end{array}\right]: \widetilde{W}_{\Delta} \rightarrow V .
$$

We solve the dual system (4.4) using the preconditioned conjugate gradient (CG) algorithm with the preconditioner

$$
M^{-1}:=B_{D, \Delta} S B_{D, \Delta}^{\top}=\sum_{i=1}^{N} D_{\Delta}^{(i)} B_{\Delta}^{(i)} S^{(i)} B_{\Delta}^{(i) \top} D_{\Delta}^{(i)} ;
$$

see Farhat et al. (2001) and Klawonn et al. (2002).

We now recall an abstract framework for the analysis of FETI-DP algorithms, which was originally given in Klawonn et al. (2002) and recalled in Toselli \& Vasseur (2004). It turns out that condition number bounds rely on one stability estimate for the following jump operator

$$
P_{\Delta}:=B_{D, \Delta}^{T} B_{\Delta}: \widetilde{W} \longrightarrow \widetilde{W} .
$$

We summarize the properties of $P_{\Delta}$ proven in Klawonn et al. (2002, Section 6) in the following lemma. LEMMA 4.1 The operator $P_{\Delta}$ is a projection and preserves the jump of any function $w \in \widetilde{W}$, i.e.

$$
B_{\Delta} P_{\Delta} \mathbf{w}=B_{\Delta} \mathbf{w}
$$

If $\mathbf{v}:=P_{\Delta} \mathbf{w}$, for $\mathbf{w} \in \widetilde{W}$, then on every fine edge $e \in \Gamma_{i, h}$, we have

$$
\mathbf{v}^{(i)}=\sum_{j \in \mathcal{N}_{e}} \delta_{j}^{\dagger}\left(\mathbf{w}^{(i)}-\mathbf{w}^{(j)}\right) .
$$

Finally, $P_{\Delta} \mathbf{w}=0$, if $\mathbf{w} \in \widehat{W}$.

The following fundamental result can be found in Klawonn et al. (2002, Theorem 1); see also Toselli \& Widlund (2004, Section 6.4.3). It employs the norms

$$
|\mathbf{v}|_{S}^{2}:=\langle\mathbf{v}, S \mathbf{v}\rangle=\sum_{i=1}^{N}\left\langle\mathbf{v}^{(i)}, S^{(i)} \mathbf{v}^{(i)}\right\rangle, \quad|\mathbf{v}|_{\widetilde{S}}^{2}:=\langle\mathbf{v}, \widetilde{S} \mathbf{v}\rangle .
$$

THEOREM 4.2 Let $C_{P_{\Delta}}$ be such that

$$
\left|P_{\Delta} \mathbf{w}_{\Delta}\right|_{S}^{2} \leqslant C_{P_{\Delta}}\left|\mathbf{w}_{\Delta}\right|_{\widetilde{S}}^{2}, \quad \mathbf{w}_{\Delta} \in \widetilde{W}_{\Delta} .
$$

Then, if $\widetilde{S}$ and $M^{-1}$ are invertible,

$$
\langle M \lambda, \lambda\rangle \leqslant\langle F \lambda, \lambda\rangle \leqslant C_{P_{\Delta}}\langle M \lambda, \lambda\rangle, \quad \lambda \in V .
$$




\section{Remarks on iterative substructuring for edge-element approximations}

Preconditioners for Schur complement systems rely on decoupling degrees of freedom associated with geometrical objects associated with subdomains, typically vertices, edges and faces for 3D continuous nodal elements; see, e.g. Dryja et al. (1994, Section 5). In our case, we only need to consider subdomain edges and faces. The performance of the corresponding preconditioned iterative method depends on how weak the coupling between the different blocks of degrees of freedom is and depends on the particular basis chosen. This decoupling may appear explicitly in the construction of finite-element subspaces as in wire basket methods, Dryja et al. (1994), but it may also be hidden in the algorithm and may not appear explicitly in the subspaces considered, as in NN or FETI methods. Indeed, the scaled matrix $B_{\Delta}$ acts on vectors of degrees of freedom and is constructed with the scaling functions $\delta_{i}^{\dagger}$. These functions are constant on the edges and faces of a subdomain and formula (4.9) naturally decouples degrees of freedom on edges and faces.

Decompositions into edge and face components are fairly harmless (i.e. logarithmically stable) operations for continuous nodal $h$ finite elements but turn out to be disastrous for edge-element approximations, as can be seen in Fig. 1 (left). More precisely, we refer to Fig. 1 (right) and consider the gradient of a continuous, scalar, piecewise trilinear function $\phi_{E}$ with vanishing nodal values on the closure of a subdomain $\Omega_{i}$ except at one node on a coarse edge $E$ where it is one. Since $\phi_{E}$ decreases linearly from one to zero along an edge of length $O(h)$, its tangential component is $O\left(h^{-1}\right)$. This vector is curl-free and has a low energy:

$$
\left\|\nabla \phi_{E}\right\|_{H\left(\operatorname{curl} ; \Omega_{i}\right)}^{2}=\left\|\nabla \phi_{E}\right\|_{L^{2}\left(\Omega_{i}\right)^{3}}^{2}=O\left(h^{-2} \cdot h^{3}\right)=O(h) .
$$

We recall that the square of the $L^{2}$ norm of a basis function is $O\left(h^{3}\right)$ while that of its curl is $O(h)$. When we put to zero the degrees of freedom on the two faces adjacent to $E$, we obtain a vector $\mathbf{w}$ with a non-vanishing curl and therefore with a much larger energy

$$
\|\mathbf{w}\|_{H\left(\operatorname{curl} ; \Omega_{i}\right)}^{2} \sim\|\operatorname{curl} \mathbf{w}\|_{L^{2}\left(\Omega_{i}\right)^{3}}^{2}=O\left(h^{-2} \cdot h\right)=O(1 / h) .
$$

The constant $C_{P_{\Delta}}$ in Theorem 4.2 is therefore expected to grow at least as $h^{-2}$, thus resulting in a condition number that grows at least as $h^{-2}$. The situation is the same as for general $p$ and $h p$ approximations, for which strong coupling exists between edge and face components, unless very special bases are employed; see, e.g. Mandel (1990).

We stress that this will happen independently of the chosen primal constraints, unless they impose the continuity of all the fine degrees of freedom across the coarse edges; in the latter case, the difference $\mathbf{w}^{(i)}-\mathbf{w}^{(j)}$ in formula (4.9) already vanishes on the coarse edges and no decoupling takes place. Any NN or FETI method which employs the standard 3D edge-element basis is bound to show a factor $h^{-2}$ in the condition number.

\section{FETI-DP algorithms for edge-element approximations}

The discussion in the previous section suggests that before devising effective FETI methods, a change of basis is necessary. We will therefore employ a local change of basis on the local spaces $X_{i}$. New basis functions are only needed for the degrees of freedom associated with the edges of the subdomains. In this new basis, it will be relatively straightforward to find a good set of primal constraints that ensure scalability and a coarse problem of relatively small size. 


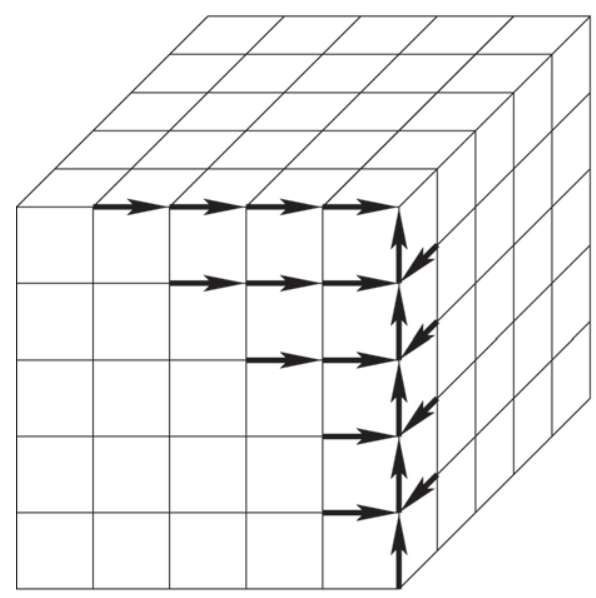

E

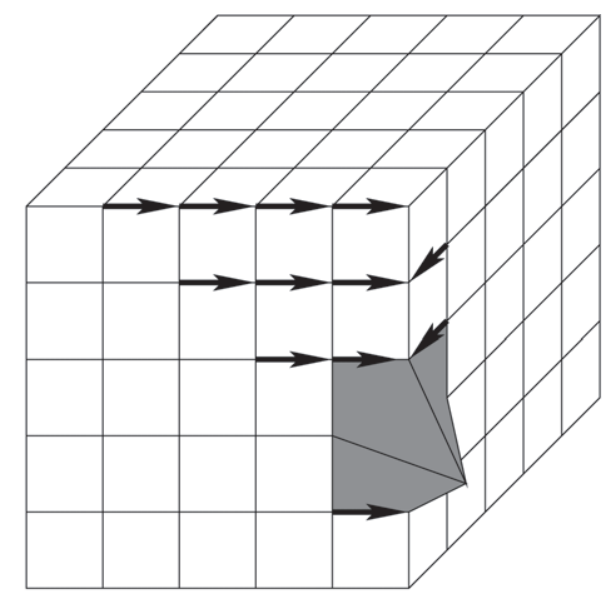

$\phi_{\mathrm{E}}$

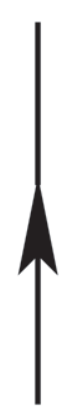

$\mathbf{w}_{\mathrm{E}}$

FIG. 1. Two types of basis functions associated with a subdomain edge: the standard basis (left) and one consisting of gradients of continuous, scalar, nodal functions associated with nodes internal to the coarse edge and one vector function with unitary tangential component along the coarse edge.

\subsection{A change of basis}

We consider a local space $X_{i}$ associated with a substructure $\Omega_{i}$ and introduce the following new basis.

DEFINITION 6.1 (New basis)

1. The basis functions associated with the interior edges $e$ in $\Omega_{i, h}$ and with those in the interior of the faces are the same as for the standard basis.

2. The basis functions associated with a subdomain edge $E$ are:

(a) one vector function $\Phi_{E}$ with unitary tangential component along $E$ and vanishing tangential component along all the fine edges $e \in \mathcal{E}_{h}$ that lie on the remaining coarse edges, on the faces and in the interior of $\Omega_{i}$;

(b) the gradients of continuous, scalar, nodal functions associated with the interior nodes of $E$; these scalar functions take the value zero at all the nodes in the closure of $\Omega_{i}$ except at one node in the interior of $E$, where they are equal to one.

This new basis is described in Fig. 1 and it has the same dimension as the old one. The degree of freedom associated with a basis function $\Phi_{E}$ is the average over the coarse edge $E$, while those associated with the basis functions at point 2(b) are values at the nodes internal to the subdomain edges. The new basis functions are introduced only for the coarse subdomain edges and it can be easily proven that they are linearly independent. The new degrees of freedom can still be partitioned into interior, face and edge. For an interface function $\mathbf{w} \in W_{i}$ they give rise to the following decomposition:

$$
\mathbf{w}=\sum_{E}\left(\mathbf{w}_{E}+\nabla_{S} \phi_{E}\right)+\sum_{F} \mathbf{w}_{F}, \quad \text { on } \partial \Omega_{i}
$$

A face component $\mathbf{w}_{F}$ vanishes on all faces except $F$ and has a vanishing tangential component along all coarse edges. The component $\mathbf{w}_{E}$ has a constant tangential component along the edge $E$ and vanishing 
degrees of freedom on all faces and remaining edges; we will refer to it in the following as constant component along the edge $E$. Finally, the scalar function $\phi_{E}$ vanishes at all the nodes except at those in the interior of $E$; we will refer to $\nabla_{S} \phi_{E}$ as the gradient component along $E$.

Given a local vector $\mathbf{u} \in X_{i}$, the corresponding vectors of degrees of freedom in the new and old bases are related by

$$
\mathbf{u}^{\text {new }}=Q_{i} \mathbf{u}^{\text {old }}, \quad \mathbf{u}^{\text {old }}=Q_{i}^{-1} \mathbf{u}^{\text {new }}=R_{i} \mathbf{u}^{\text {new }} .
$$

The matrix $R_{i}$ can easily be found by noting that its columns are associated with the new basis functions and the entries of each column are the degrees of freedom in the old basis associated with one new basis function.

Definition 6.2 (Change of basis) The matrix $R_{i}$ is defined as follows:

1. Columns for the new functions associated with the degrees of freedom in the interior of $\Omega_{i}$ or in the interior of a face (cf. point 1 in Definition 6.1) consist of zeros except for one entry corresponding to a fine edge where they take the value 1 .

2. The column for a basis function that has unit tangential component along a coarse edge $E$ (cf. point 2(a) in Definition 6.1) is zero except for the entries relative to the fine edges $e \subset E$, where it takes the value 1 or -1 , depending on the convention chosen for the direction of $e$ and $E$.

3. The column for the gradient of a scalar nodal function associated with a node $x_{k}$ on an edge $E$ (cf. point 2(b) in Definition 6.1) is zero except for the entries relative to the fine edges $e \subset \bar{\Omega}_{i}$ that have $x_{k}$ as an end-point; these entries are equal to $\pm|e|^{-1}$ depending on the convention chosen for the direction of $e$.

We note that $R_{i}$ has very few non-zero entries and its inverse is also sparse. The new basis and the definition of the matrix $R_{i}$ do not rely on the fact that the substructures are elements of a coarse mesh or have a special shape, but can straightforwardly be defined for less regular subdomains produced by e.g. practical mesh partitioners.

\subsection{Primal constraints}

We consider the FETI-DP algorithm introduced in Section 4. We assume from now on that vectors of primal degrees of freedom are relative to the new basis introduced in the previous section and recall that we still have a partition into edge and face degrees of freedom. The matrix $B_{\Delta}$ still consists of zeros and ones, the scaling functions are defined by associating with each new degree of freedom the ratio in (4.6), which only depends on the subdomain partition and the coefficients $A$ or $B$, and the Schur complements are those obtained from stiffness matrices in the new basis.

We are only left with the definition of suitable primal constraints. Our choices are related to the decomposition (6.1) of the degrees of freedom for the new basis. We first impose that, for each coarse edge of the interface $\Gamma$, the degree of freedom associated with the term $\mathbf{w}_{E}$ in the decomposition (6.1) is the same for each subdomain that contains $E$. The degree of freedom associated with this term is the average of the tangential component along the edge $E$ :

$$
a_{E}(\mathbf{w})=a_{E}(w):=|E|^{-1} \int_{E} \mathbf{w} \cdot \mathbf{t}_{E} \mathrm{~d} s=|E|^{-1} \int_{E} w \mathrm{~d} s .
$$

DEFINITION 6.3 (Algorithm A) The space $\widetilde{W}=\widetilde{W}_{A}$ is the subspace of $W$ of interface vectors for which the averages $a_{E}(\mathbf{w})$ of the tangential component along the coarse edges $E$ are equal, independent of which component of $\mathbf{w} \in \widetilde{W}_{A}$ is used in the evaluation of these averages. The local spaces $\widetilde{W}_{\Delta, i}$ 
consist of vectors with a vanishing average for the tangential component along the edges, while the primal subspace $\widehat{W}_{\Pi}$ consists of continuous vectors with a constant tangential component along each coarse edge and zero components $\mathbf{w}_{F}$ and $\nabla_{S} \phi_{E}$ in the decomposition (6.1).

We note that, in case the subdomain partition coincides with a coarse mesh, the primal constraints are the degrees of freedom for the edge-element coarse space. Algorithm A is not, however, expected to perform well. Indeed, we see that there are no constraints acting on the scalar functions $\phi_{E}$ in the decomposition (6.1), except from the requirement that they vanish at the end-points of the edges $E$. We therefore expect that Algorithm A performs at least as badly as the corresponding FETI-DP for continuous, scalar finite elements where only primal constraints at the subdomain vertices are imposed; cf. Algorithm A in Klawonn et al. (2002).

Together with averages along the edges, we need to add additional constraints involving the gradient components along the edges. Looking at which algorithms are effective for scalar approximations, averages of the scalar functions $\phi_{E}$

$$
\left\langle\phi_{E}\right\rangle_{E}:=|E|^{-1} \int_{E} \phi_{E} \mathrm{~d} s,
$$

along the subdomain edges should be imposed to be continuous as well; see Algorithm C in Klawonn et al. (2002) and Toselli \& Widlund (2004, Section 6.4.2).

Before giving a precise definition, we want to express these second averages in terms of the vector $\mathbf{w} \in W_{i}$ itself. We assume that the vector $\mathbf{w}$ in (6.1) has a vanishing average (6.3) for the tangential component along an edge $E$. We denote by $s$ the arclength along $E$. Since $\phi_{E}$ vanishes at the endpoints of $E$, integration by parts yields

$$
\begin{aligned}
\left\langle\phi_{E}\right\rangle_{E} & =|E|^{-1} \int_{E} \phi_{E} \mathrm{~d} s=-|E|^{-1} \int_{E} \phi_{E}^{\prime} s \mathrm{~d} s=-|E|^{-1} \int_{E}\left(\nabla \phi_{E} \cdot \mathbf{t}_{E}\right) s \mathrm{~d} s \\
& =-|E|^{-1} \int_{E}\left(\mathbf{w}-\mathbf{w}_{E}\right) \cdot \mathbf{t}_{E} s \mathrm{~d} s=-|E|^{-1} \int_{E}\left(\mathbf{w} \cdot \mathbf{t}_{E}\right) s \mathrm{~d} s .
\end{aligned}
$$

When $\mathbf{w} \cdot \mathbf{t}_{E}$ has a vanishing mean value, the averages of the functions $\phi_{E}$ are therefore equal to firstorder moments of the original vector $\mathbf{w}$ along the edge. For each coarse edge $E$, we define the continuous, scalar function $\theta_{E}$ to be zero at all the nodes of the interface $\Gamma$ except at those internal to $E$ where it is equal to one.

Definition 6.4 (Algorithm B) The space $\widetilde{W}=\widetilde{W}_{B}$ is the subspace of $W$ of interface vectors for which the averages $a_{E}(\mathbf{w})$ and $\left\langle\phi_{E}\right\rangle_{E}$ along the coarse edges, or, equivalently, $a_{E}(\mathbf{w})$ and the first-order moments

$$
|E|^{-1} \int_{E}\left(\mathbf{w} \cdot \mathbf{t}_{E}\right) s \mathrm{~d} s,
$$

are equal, independent of which component of $\mathbf{w} \in \widetilde{W}_{B}$ is used in the evaluation of these averages. The local spaces $\widetilde{W}_{\Delta, i}$ consist of vectors with vanishing average and first-order moment for the tangential component along the edges, while the primal subspace $\widehat{W}_{\Pi}$ is spanned by the same functions as for Algorithm $\mathrm{A}$ and, in addition, the gradients $\nabla_{S} \theta_{E}$ associated with the coarse edges.

Algorithm B will provide a very favorable bound. We note that, despite the difficulty of this problem, the size of the global problem that needs to be solved (equal to the number of primal constraints) is very reasonable: we have two constraints per coarse edge. In the case, for instance, where the substructures 
are elements of a coarse, cubical, uniform mesh, each subdomain has 12 edges, which is shared by four substructures. We have therefore six coarse degrees of freedom for each substructure.

We finally define an algorithm with a very large coarse space.

Definition 6.5 (Algorithm C) The space $\widetilde{W}=\widetilde{W}_{C}$ is the subspace of $W$ of interface vectors for which the tangential component along the fine edges on the coarse edges

$$
\lambda_{e}(\mathbf{w})=|e|^{-1} \int_{e} \mathbf{w} \cdot \mathbf{t}_{e} \mathrm{~d} s, \quad e \subset E,
$$

are equal. The local spaces $\widetilde{W}_{\Delta, i}$ consist of vectors with a vanishing tangential component along the edges, while the primal subspace $\widehat{W}_{\Pi}$ is spanned by the continuous basis functions associated with the fine edges that lie on a coarse edge.

A practical implementation of FETI-DP algorithms for problem (1.1) is given in Toselli \& Vasseur (2004, Section 6). While one should in principle employ matrices and vectors associated with the new basis for Algorithms A and B, it is possible to employ the original operators in the standard basis and work with the local matrices $Q_{i}$ in (6.2). We note in particular that primal constraints can be expressed in terms of the old basis and the matrix $B_{\Delta}$, which evaluates the difference between corresponding degrees of freedom, and is the same for the two bases in case, for instance, the same orientation for the corresponding fine edges is employed on different subdomains.

\section{Technical tools}

In this section, we will prove some decomposition and comparison results for edge-element vectors. Some of the ideas of this analysis have been suggested in studies of A. Buffa (personal communication). Many of our estimates depend on a logarithmic factor

$$
\omega:=1+\log (H / h)
$$

where the ratio $H / h$ is a shorthand notation for the maximum over the substructures of $H_{i} / h_{i}$. As is often customary in the analysis of iterative substructuring methods, we require that the substructures are elements of a shape-regular coarse mesh $\mathcal{T}_{H}$ or that they are a union of a uniformly bounded number of coarse shape-regular elements.

\subsection{Trace spaces}

For a substructure $\Omega_{i}$, we need to consider tangential traces of functions in $H$ (curl; $\Omega_{i}$ ) and the corresponding edge-element spaces on the boundary, on a face $F$ or along $\partial F$. We refer to e.g. Alonso \& Valli (1996) and Buffa \& Ciarlet (2001a,b) for more details and definitions.

In the following, Sobolev spaces of vectors on $\partial \Omega_{i}$ and $F$ are always understood as spaces of tangential vectors; we will use a notation with bold letters for them. We define

$$
\mathbf{H}_{\perp}^{-1 / 2}\left(\operatorname{curl}_{S} ; \partial \Omega_{i}\right)=\left\{\mathbf{u} \in \mathbf{H}_{\perp}^{-1 / 2}\left(\partial \Omega_{i}\right), \operatorname{curl}_{S} \mathbf{u} \in H^{-1 / 2}\left(\partial \Omega_{i}\right)\right\} .
$$

The space $H^{-1 / 2}\left(\partial \Omega_{i}\right)$ is the dual of $H^{1 / 2}\left(\partial \Omega_{i}\right)$, while $\mathbf{H}_{\perp}^{-1 / 2}\left(\partial \Omega_{i}\right)$ is the dual of $\mathbf{H}_{\perp}^{1 / 2}\left(\partial \Omega_{i}\right)$. The precise definitions of $\mathbf{H}_{\perp}^{1 / 2}\left(\partial \Omega_{i}\right)$ and its norm are given in Appendix A since they are not important at this stage: it is, roughly speaking, the space of tangential vectors on $\partial \Omega_{i}$, the restriction of which to 
a face $F$ belongs to $\mathbf{H}^{1 / 2}(F)$ with a normal component along the face boundaries in $H^{1 / 2}\left(\partial \Omega_{i}\right)$; see Alonso \& Valli (1996) and Buffa \& Ciarlet (2001a,b).

For a face $F$, we define

$$
\mathbf{H}_{\perp, 00}^{-1 / 2}\left(\operatorname{curl}_{S} ; F\right)=\left\{\mathbf{u} \in \mathbf{H}_{\perp, 00}^{-1 / 2}(F), \operatorname{curl}_{S} \mathbf{u} \in H_{00}^{-1 / 2}(F)\right\},
$$

where $\mathbf{H}_{\perp, 00}^{-1 / 2}(F)$ and $H_{00}^{-1 / 2}(F)$ are the duals of $\mathbf{H}_{\perp, 00}^{1 / 2}(F)$ and $H_{00}^{1 / 2}(F)$, respectively, consisting of functions in $\mathbf{H}^{1 / 2}(F)$ and $H^{1 / 2}(F)$ for which the extensions by zero to $\partial \Omega_{i}$ belong to $\mathbf{H}_{\perp}^{1 / 2}\left(\partial \Omega_{i}\right)$ and $H^{1 / 2}\left(\partial \Omega_{i}\right)$, respectively. If $\mathcal{E} \mathbf{u}$ and $\mathcal{E} \phi$ denote these extensions, we employ the norms

$$
\|\mathbf{u}\|_{\mathbf{H}_{\perp, 00}^{1 / 2}(F)}=\|\mathcal{E} \mathbf{u}\|_{\mathbf{H}_{\perp}^{1 / 2}\left(\partial \Omega_{i}\right)}, \quad\|\phi\|_{H_{00}^{1 / 2}(F)}=\|\mathcal{E} \phi\|_{H^{1 / 2}\left(\partial \Omega_{i}\right)}
$$

The space $\mathbf{H}^{-1 / 2}\left(\operatorname{curl}_{S} ; F\right)$ is defined in a similar way:

$$
\mathbf{H}^{-1 / 2}\left(\operatorname{curl}_{S} ; F\right)=\left\{\mathbf{u} \in \mathbf{H}^{-1 / 2}(F), \operatorname{curl}_{S} \mathbf{u} \in H^{-1 / 2}(F)\right\} .
$$

The spaces $\mathbf{H}_{\perp}^{-1 / 2}\left(\operatorname{curl}_{S} ; \partial \Omega_{i}\right), \mathbf{H}_{\perp, 00}^{-1 / 2}\left(\operatorname{curl}_{S} ; F\right)$ and $\mathbf{H}^{-1 / 2}\left(\operatorname{curl}_{S} ; F\right)$ are equipped with the graph norms. We note that $H^{-1 / 2}(F), \mathbf{H}^{-1 / 2}(F)$ and $\mathbf{H}^{-1 / 2}\left(\operatorname{curl}_{S} ; F\right)$ are proper subspaces of $H_{00}^{-1 / 2}(F)$, $\mathbf{H}_{\perp, 00}^{-1 / 2}(F)$ and $\mathbf{H}_{\perp, 00}^{-1 / 2}\left(\operatorname{curl}_{S} ; F\right)$, respectively. In addition, by simple computation,

$$
\begin{aligned}
& \|\phi\|_{H_{00}^{-1 / 2}(F)} \leqslant C\|\mathcal{E} \phi\|_{H^{-1 / 2}\left(\partial \Omega_{i}\right)} \leqslant C\|\phi\|_{H^{-1 / 2}(F)}, \\
& \|\mathbf{w}\|_{\mathbf{H}_{\perp, 00}^{-1 / 2}(F)} \leqslant C\|\mathcal{E} \mathbf{w}\|_{\mathbf{H}_{\perp}^{-1 / 2}\left(\partial \Omega_{i}\right)} \leqslant C\|\mathbf{w}\|_{\mathbf{H}^{-1 / 2}(F)} .
\end{aligned}
$$

In the following, we will also employ $H^{-1}(\partial F)$, the dual of $H^{1}(\partial F)$.

Throughout, we will work with scaled norms for the spaces $H^{s}(\mathcal{D}), s>0$, obtained from the definition of the Sobolev norm on a region with diameter one and a dilation. Thus, if $H_{\mathcal{D}}$ is the diameter of a region $\mathcal{D} \subset \mathbb{R}^{n}$, we define

$$
\|u\|_{H^{1}(\mathcal{D})}^{2}=|u|_{H^{1}(\mathcal{D})}^{2}+H_{\mathcal{D}}^{-2}\|u\|_{L^{2}(\mathcal{D})}^{2},
$$

for a substructure $\mathcal{D}=\Omega_{i}$ or the boundary of one of its faces $\mathcal{D}=\partial F$, and

$$
\|u\|_{H^{1 / 2}(\mathcal{D})}^{2}=|u|_{H^{1 / 2}(\mathcal{D})}^{2}+H_{\mathcal{D}}^{-1}\|u\|_{L^{2}(\mathcal{D})}^{2},
$$

for the boundary of a substructure $\mathcal{D}=\partial \Omega_{i}$ or one of its faces $\mathcal{D}=F$. Analogous definitions hold for the spaces of vectors. The definition of dual norms employs these scaled norms. As is standard in the analysis of DD methods, inequalities are obtained for regions of unit diameter and then by a scaling argument that provides an explicit dependence on the diameter of the regions.

We have the following trace estimates. The first two are a straightforward consequence of the fact that curl $\mathbf{u} \in L^{2}\left(\Omega_{i}\right)^{3}$ has a vanishing divergence and therefore its normal component is well defined; see, e.g. Brezzi \& Fortin (1991, Lemma 1.2, Chapter III). The others can be found in, e.g. Buffa \& Ciarlet (2001b), Theorems 4.6 and 6.6 and Proposition 6.3. 
LEMMA 7.1 Let $\Omega_{i}$ be a substructure and $F$ one of its faces. Then, there exists a constant $C$, independent of $H_{i}$, such that, for $\mathbf{u} \in H\left(\operatorname{curl} ; \Omega_{i}\right)$,

$$
\begin{gathered}
\left\|\operatorname{curl}_{S} \mathbf{u}\right\|_{H^{-1 / 2}\left(\partial \Omega_{i}\right)}^{2} \leqslant C\|\operatorname{curl} \mathbf{u}\|_{L^{2}\left(\Omega_{i}\right)}^{2}, \\
\left\|\operatorname{curl}_{S} \mathbf{u}\right\|_{H_{00}^{-1 / 2}(F)}^{2} \leqslant C\|\operatorname{curl} \mathbf{u}\|_{L^{2}\left(\Omega_{i}\right)}^{2}, \\
\left\|\mathbf{u}_{\mathbf{H}_{\perp}^{-1 / 2}\left(\partial \Omega_{i}\right)}^{2}+H_{i}^{2}\right\| \operatorname{curl}_{S} \mathbf{u} \|_{H^{-1 / 2}\left(\partial \Omega_{i}\right)}^{2} \\
\|\mathbf{u}\|_{\mathbf{H}_{\perp, 00}^{-1 / 2}(F)}^{2}+H_{i}^{2}\left\|\operatorname{curl}_{S} \mathbf{u}\right\|_{H_{00}^{-1 / 2}(F)}^{2} \leqslant C\left(\|\mathbf{u}\|_{L^{2}\left(\Omega_{i}\right)}^{2}+H_{i}^{2}\|\operatorname{curl} \mathbf{u}\|_{L^{2}\left(\Omega_{i}\right)}^{2}\right), \\
\left.\left\|_{L^{2}\left(\Omega_{i}\right)}^{2}+H_{i}^{2}\right\| \operatorname{curl} \mathbf{u} \|_{L^{2}\left(\Omega_{i}\right)}^{2}\right) .
\end{gathered}
$$

In addition, for $\mathbf{u} \in \mathbf{H}^{-1 / 2}\left(\operatorname{curl}_{S} ; F\right)$, its tangential component $u=\mathbf{u} \cdot \mathbf{t}_{\partial F}$ satisfies

$$
\|u\|_{H^{-1}(\partial F)}^{2} \leqslant C\left(\|\mathbf{u}\|_{\mathbf{H}^{-1 / 2}(F)}^{2}+H_{i}^{2}\left\|\operatorname{curl}_{S} \mathbf{u}\right\|_{H^{-1 / 2}(F)}^{2}\right) .
$$

We note that tangential components along face boundaries are not defined for every vector in $H\left(\right.$ curl; $\left.\Omega_{i}\right)$ or its appropriate trace space. They can be defined, however, for edge-element vectors and the link between the stronger and weaker norms is given by the inverse inequalities of the following lemma.

LEMMA 7.2 Let $\Omega_{i}$ be a substructure and $F$ one of its faces and assume that the mesh on $F$ is quasiuniform. Let $\mathbf{u} \in W_{i}$. Then there exists a constant, independent of $h$ and $H_{i}$, such that

$$
\begin{gathered}
\left\|\operatorname{curl}_{S} \mathbf{u}\right\|_{H^{-1 / 2}(F)}^{2} \leqslant C \omega^{2}\left\|\operatorname{curl}_{S} \mathbf{u}\right\|_{H^{-1 / 2}\left(\partial \Omega_{i}\right)}^{2}, \\
\|\mathbf{u}\|_{\mathbf{H}^{-1 / 2}(F)}^{2} \leqslant C \omega^{2}\|\mathbf{u}\|_{\mathbf{H}_{\perp}^{-1 / 2}\left(\partial \Omega_{i}\right)}^{2}, \\
\|\mathbf{u}\|_{\mathbf{H}^{-1 / 2}(F)}^{2}+H_{i}^{2}\left\|\operatorname{curl}_{S} \mathbf{u}\right\|_{H^{-1 / 2}(F)}^{2} \leqslant C \omega^{2}\left(\|\mathbf{u}\|_{\mathbf{H}_{\perp}^{-1 / 2}\left(\partial \Omega_{i}\right)}^{2}+H_{i}^{2}\left\|\operatorname{curl}_{S} \mathbf{u}\right\|_{H^{-1 / 2}\left(\partial \Omega_{i}\right)}^{2}\right),
\end{gathered}
$$

with $\omega$ the logarithmic factor defined in (7.1).

We note that a stronger version of the first inequality, involving a piecewise constant function on $\partial \Omega_{i}$, was already proven in Wohlmuth (2000, Lemma 4.4) by using an equivalent dual norm for $H^{-1 / 2}\left(\partial \Omega_{i}\right)$ with a supremum taken over a finite-element space of piecewise bilinear, continuous functions augmented with one bubble for each boundary element. That proof, however, cannot be easily generalized to the edge-element tangential space in $\mathbf{H}_{\perp}^{-1 / 2}\left(\partial \Omega_{i}\right)$. The lemma is proven in Appendix A, using a localization result and an inverse inequality for norms in Sobolev spaces with negative exponents.

\subsection{Decompositions associated with the edges}

For a substructure $\Omega_{i}$, we now consider the decomposition (6.1) in more detail. The edge contributions can be found in the following way:

We consider $\mathbf{w} \in W_{i}$ and an edge $E \subset \partial F, F \subset \partial \Omega_{i}$. We assume that the edge tangent vectors $\mathbf{t}_{E}$ have the same direction as $\mathbf{t}_{\partial F}$. Let $w$ be its tangential component along $\partial F$. We have

$$
\mathbf{w}_{E}=a_{E}(\mathbf{w}) \Phi_{E},
$$

or, equivalently,

$$
w_{E}=\mathbf{w}_{E} \cdot \mathbf{t}_{E}=a_{E}(\mathbf{w})=a_{E}(w) .
$$


Here, $\Phi_{E}$ is the basis function of point 2(a), Definition 6.1. Let $\Phi_{E}(s)$ be the function that is one on $E$ and zero on the rest of $\partial F$. By definition, we have

$$
\int_{E}\left(w-w_{E}\right) \mathrm{d} s=\int_{E}\left(w-a_{E}(w)\right) \mathrm{d} s=0 .
$$

If $s \in[0,|E|]$ is the arclength along $E$, there then exists a continuous, piecewise linear scalar function $\phi_{E}(s)$, vanishing on $\partial F \backslash E$, such that

$$
\phi_{E}^{\prime}(s)=w(s)-a_{E}(w), \quad \phi_{E}(s)=\int_{0}^{s}\left(w\left(s^{\prime}\right)-a_{E}(w)\right) \mathrm{d} s^{\prime}, \quad \text { on } E .
$$

We have therefore the decomposition

$$
w=\sum_{E \subset \partial F}\left(a_{E}(w) \Phi_{E}+\phi_{E}^{\prime}\right), \quad \text { on } \partial F
$$

which gives the tangential component along $\partial F$ of the decomposition (6.1). A function $\phi_{E}$ defined on the whole of $\Omega_{i}$ can then be obtained as a zero extension, by setting to zero all the nodal values that do not lie inside $E$. We have therefore found the terms $\nabla \phi_{E}$ in the decomposition (6.1).

We note that the decomposition (7.3) is not logarithmically stable. Roughly speaking, $a_{E}(w)$ is not a bounded linear functional in $H^{-1}(\partial F)$, the appropriate space of the tangential component $w$ along $\partial F$ (apart from a logarithmic factor). For this reason, we are forced to consider a second decomposition. We define the average

$$
a_{\partial F}(\mathbf{w})=a_{\partial F}(w):=|\partial F|^{-1} \int_{\partial F} w \mathrm{~d} s .
$$

There then exists a continuous, piecewise linear scalar function $\phi_{\partial F}(s)$, such that

$$
\phi_{\partial F}^{\prime}(s)=w(s)-a_{\partial F}(w), \quad \phi_{\partial F}(s)=\int_{0}^{s}\left(w\left(s^{\prime}\right)-a_{\partial F}(w)\right) \mathrm{d} s^{\prime}+C, \quad \text { on } \partial F .
$$

This gives the second decomposition

$$
w=a_{\partial F}(w)+\phi_{\partial F}^{\prime}, \quad \text { on } \partial F .
$$

We note that $\phi_{\partial F}$ does not necessarily vanish at all the vertices on $\partial F$ and is defined up to an additive constant. Once this constant is fixed and a suitable extension to $F$ is defined, the corresponding edgeelement function $\nabla \phi_{\partial F}$ provides an extension of the tangential component $\phi_{\partial F}^{\prime}$ inside $\Omega_{i}$. The terms associated with the two decompositions are illustrated in Fig. 2 for the case of a triangular face. We make the following choice.

DeFinition 7.3 The constant in (7.4) is chosen such that the scalar function $\phi_{\partial F}$ has a vanishing mean value $\left\langle\phi_{\partial F}\right\rangle_{E}$ over one of the edges $E \subset \partial F$; $\phi_{\partial F}$ is then extended by zero to all the remaining nodes in $\bar{\Omega}_{i} \backslash \partial F$.

A more intuitive and equivalent definition of $\phi_{\partial F}$ is the following: we consider the function

$$
\phi_{\partial F}(s)=\int_{0}^{s}\left(w\left(s^{\prime}\right)-a_{\partial F}(w)\right) \mathrm{d} s^{\prime}, \quad s \in[0,|\partial F|],
$$



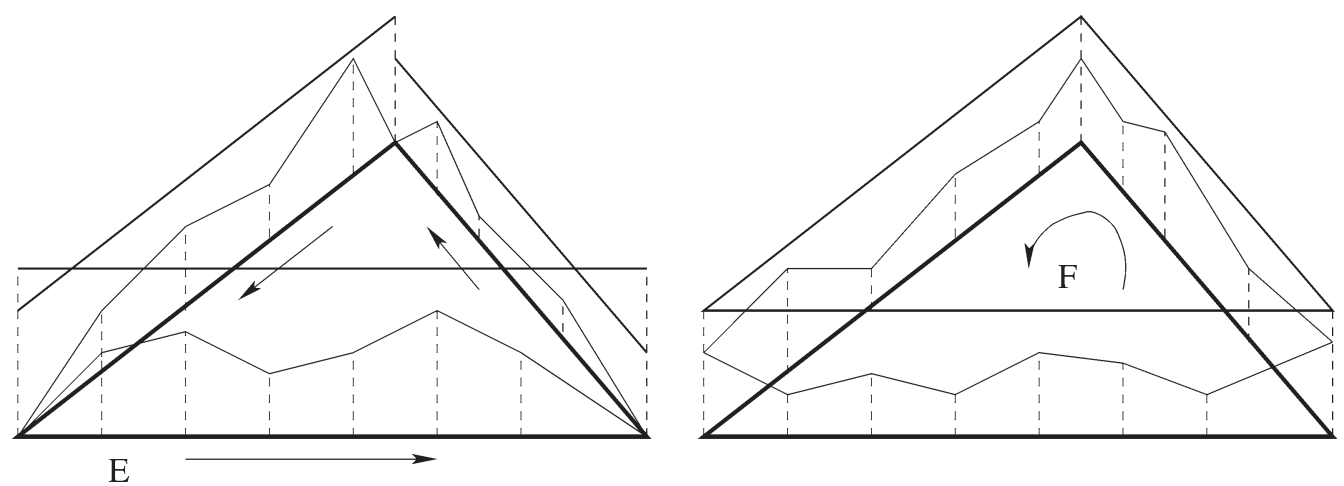

FIG. 2. Terms associated with two decompositions of the tangential component $w$ along the boundary of a face: $\left\{a_{E}(w) \Phi_{E}, \phi_{E}\right\}$ for (7.3) (left) and $a_{\partial F}(w)$ and $\phi_{\partial F}$ for (7.5) (right).

defined on $\partial F$ and vanishing at one of the vertices. We then take its mean value along an edge $E$ and assign this value at all the internal nodes on $F$ and the remaining ones in $\bar{\Omega}_{i}$. This is related to the similar coarse interpolant in Dryja et al. (1994, Section 6.2), where averages along face boundaries are employed. Since only the gradient of this function has a meaning here, we can subtract the edge average on $\bar{\Omega}_{i}$ and obtain the function in Definition 7.3. Here, we have chosen to work with an edge average instead of one on $\partial F$ since for the proof of Lemma 8.2 we will need to compare gradient contributions for two different substructures that may only have an edge in common but not an entire face.

We now consider the decomposition (7.5) in more detail and show that it is logarithmically stable. The following is a trivial property of the zero extension from a subdomain edge; see Dryja et al. (1994, Lemma 4.7).

LEMMA 7.4 Let $\Omega_{i}$ be a substructure and $F$ one of its faces. If $\phi$ is a continuous, piecewise trilinear function that vanishes at all nodes in $\bar{\Omega}_{i}$ except those on $\partial F$, then

$$
\|\nabla \phi\|_{L^{2}\left(\Omega_{i}\right)}^{2} \leqslant C\|\phi\|_{L^{2}(\partial F)}^{2} .
$$

We next find a bound for the face average.

Lemma 7.5 Let $\Omega_{i}$ be a substructure and $F$ one of its faces. There exists a constant, independent of $H_{i}$ and the mesh size, such that, for $\mathbf{w} \in X_{i}$,

$$
\left|a_{\partial F}(w)\right|^{2} \leqslant C H_{i}^{-1} \omega^{2}\|\mathbf{c u r l} \mathbf{w}\|_{L^{2}\left(\Omega_{i}\right)}^{2} .
$$

Proof. We have

$$
\begin{aligned}
\left|a_{\partial F}(w)\right| & =|\partial F|^{-1}\left|\int_{\partial F} \mathbf{w} \cdot \mathbf{t}_{\partial F} \mathrm{~d} s\right|=|\partial F|^{-1}\left|\int_{F} \operatorname{curl}_{S} \mathbf{w} \mathrm{d} S\right| \\
& \leqslant C H_{i}^{-1}\left\|\operatorname{curl}_{S} \mathbf{w}\right\|_{H^{-1 / 2}(F)}\|1\|_{H^{1 / 2}(F)} .
\end{aligned}
$$

The proof is concluded by using the first inequality of Lemma 7.2, the trace estimate in Lemma 7.1 and the fact that $\|1\|_{H^{1 / 2}(F)} \leqslant C \sqrt{H_{i}}$.

We note that a better bound can be proven; see the proof of Wohlmuth (2000, Lemma 4.1). Here, we chose a different proof that relies on Lemma 7.2, in order to give a self-contained presentation. Indeed, there is no difference in our final result. 
We now need the following technical result.

LEMMA 7.6 Let $F$ be a face of a substructure and $E \subset \partial F$ one of its edges. If $\phi$ is a continuous, piecewise linear function on $\partial F$ and $\langle\phi\rangle_{E}$ its mean value on $E$, then

$$
\left\|\phi-\langle\phi\rangle_{E}\right\|_{L^{2}(\partial F)} \leqslant C\left\|\phi^{\prime}\right\|_{H^{-1}(\partial F)}
$$

Proof. We have, for an arbitrary constant $c$,

$$
\left\|\phi-\langle\phi\rangle_{E}\right\|_{L^{2}(\partial F)}=\left\|(\phi-c)-\langle\phi-c\rangle_{E}\right\|_{L^{2}(\partial F)} \leqslant C\|\phi-c\|_{L^{2}(\partial F)} .
$$

Choosing $c=\langle\phi\rangle_{\partial F}$, the mean value over $\partial F$, therefore, gives

$$
\begin{aligned}
& \| \phi-\langle\phi\rangle_{E}\left\|_{L^{2}(\partial F)} \leqslant C\right\| \phi-\langle\phi\rangle_{\partial F} \|_{L^{2}(\partial F)} \\
&=C \sup _{\psi \in L^{2}(\partial F)} \frac{\int_{\partial F}\left(\phi-\langle\phi\rangle_{\partial F}\right) \psi \mathrm{d} s}{\|\psi\|_{L^{2}(\partial F)}}=C \sup _{\psi \in L^{2}(\partial F)} \frac{\int_{\partial F} \phi\left(\psi-\langle\psi\rangle_{\partial F}\right) \mathrm{d} s}{\|\psi\|_{L^{2}(\partial F)}} \\
& \leqslant C \sup _{\psi \in L^{2}(\partial F)} \frac{\int_{\partial F} \phi\left(\psi-\langle\psi\rangle_{\partial F}\right) \mathrm{d} s}{\left\|\psi-\langle\psi\rangle_{\partial F}\right\|_{L^{2}(\partial F)}}=C \sup _{\substack{\eta \in H^{1}(\partial F) \\
\eta(0)=0}} \frac{\int_{\partial F} \phi \eta^{\prime} \mathrm{d} s}{|\eta|_{H^{1}(\partial F)}} \\
&=C \sup _{\substack{\eta \in H^{1}(\partial F) \\
\eta(0)=0}} \frac{-\int_{\partial F} \phi^{\prime} \eta \mathrm{d} s}{|\eta|_{H^{1}(\partial F)}} \leqslant C\left\|\phi^{\prime}\right\|_{H^{-1}(\partial F)} .
\end{aligned}
$$

REMARK 7.7 We note that it is necessary to subtract an average from $\phi$ for the previous lemma, in order to obtain a good bound; see also Definition 7.3 and Lemma 7.8 below. If $P$ is a node on $\partial F$, indeed we only have

$$
\|\phi-\phi(P)\|_{L^{2}(\partial F)}^{2} \leqslant C(H / h)\left\|\phi^{\prime}\right\|_{H^{-1}(\partial F)}^{2} .
$$

This bound can be obtained by using a similar reasoning and the trivial bound for a linear function on a small edge $e \subset \partial F:\|\phi\|_{L^{\infty}(e)}^{2} \leqslant(C / h)\|\phi\|_{L^{2}(e)}^{2}$.

We now give a discrete trace estimate for a face boundary.

LEMMA 7.8 Let $F$ be a face of a substructure $\Omega_{i}$ and $\mathbf{w} \in W_{i}$. If $\phi_{\partial F}$ is chosen according to Definition 7.3, then there exists a constant, independent of $H_{i}$ and the mesh size, such that

$$
\left\|\phi_{\partial F}\right\|_{L^{2}(\partial F)}^{2} \leqslant C \omega^{2}\left(\|\mathbf{w}\|_{L^{2}\left(\Omega_{i}\right)}^{2}+H_{i}^{2}\|\mathbf{c u r l} \mathbf{w}\|_{L^{2}\left(\Omega_{i}\right)}^{2}\right) .
$$

Proof. We recall that Definition 7.3 ensures that the mean value $\left\langle\phi_{\partial F}\right\rangle_{E}$ vanishes for an edge $E \subset \partial F$. Using Lemma 7.6 and (7.5), we can then write

$$
\begin{aligned}
\left\|\phi_{\partial F}\right\|_{L^{2}(\partial F)} & \leqslant\left\|\phi_{\partial F}^{\prime}\right\|_{H^{-1}(\partial F)}=\left\|w-a_{\partial F}(w)\right\|_{H^{-1}(\partial F)} \\
& \leqslant\|w\|_{H^{-1}(\partial F)}+\left\|a_{\partial F}(w)\right\|_{H^{-1}(\partial F)} .
\end{aligned}
$$


The first term can be bounded using the trace estimates in Lemma 7.1 and the inverse inequalities in Lemma 7.2, while the second using Lemma 7.5 and the fact that $\|1\|_{H^{-1}(\partial F)} \leqslant C H_{i}^{3 / 2}$ since we employ scaled norms.

Bounds for the gradient component associated with $\partial F$ can then be found.

Lemma 7.9 Let $F$ be a face of a substructure $\Omega_{i}$ and $\mathbf{w} \in X_{i}$. If $\phi_{\partial F}$ is chosen according to Definition 7.3 , then there is a constant, independent of $H_{i}$ and the mesh size, such that

$$
\begin{aligned}
\left\|\nabla \phi_{\partial F}\right\|_{L^{2}\left(\Omega_{i}\right)}^{2} & \leqslant C \omega^{2}\left(\|\mathbf{w}\|_{L^{2}\left(\Omega_{i}\right)}^{2}+H_{i}^{2}\|\operatorname{curl} \mathbf{w}\|_{L^{2}\left(\Omega_{i}\right)}^{2}\right), \\
\left\|\nabla_{S} \phi_{\partial F}\right\|_{\mathbf{H}_{\perp, 00}^{-1 / 2}(F)}^{2} & \leqslant C \omega^{2}\left(\|\mathbf{w}\|_{L^{2}\left(\Omega_{i}\right)}^{2}+H_{i}^{2}\|\operatorname{curl} \mathbf{w}\|_{L^{2}\left(\Omega_{i}\right)}^{2}\right), \\
\left\|\nabla_{S} \phi_{\partial F}\right\|_{\mathbf{H}^{-1 / 2}(F)}^{2} & \leqslant C \omega^{4}\left(\|\mathbf{w}\|_{L^{2}\left(\Omega_{i}\right)}^{2}+H_{i}^{2}\|\operatorname{curl} \mathbf{w}\|_{L^{2}\left(\Omega_{i}\right)}^{2}\right) .
\end{aligned}
$$

Proof. The first bound is a straightforward application of Lemmas 7.4 and 7.8. The second can be then found thanks to the third trace estimate in Lemma 7.1 and the third by then using the second inverse inequality in Lemma 7.2.

We are only left with the task of associating a tangential vector to the average $a_{\partial F}(w)$. If $\Omega_{i}$ is an element of a coarse triangulation, this can be done by considering the coarse, edge-element basis functions in $N D\left(\Omega_{i}\right)$ associated with the edges $E \subset \partial F$ and associate with them the degree of freedom $a_{\partial F}(w)$ :

$$
\mathbf{w}_{\partial F}:=a_{\partial F}(w) \sum_{E \subset \partial F} \Phi_{E}^{H}=: a_{\partial F}(w) \Phi_{\partial F}^{H} ;
$$

see Fig. 2 for a triangular face. The generalization to the case where $\Omega_{i}$ is the union of coarse elements is straightforward. The following lemma relies on Lemma 7.5 and a scaling argument.

LEMmA 7.10 Let $\Omega_{i}$ be a substructure and $F$ one of its faces. There exists a constant, independent of $H_{i}$ and the mesh size, such that, for $\mathbf{w} \in X_{i}$,

$$
\left\|\mathbf{w}_{\partial F}\right\|_{\mathbf{H}^{-1 / 2}(F)}^{2}+H_{i}^{2}\left\|\operatorname{curl}_{S} \mathbf{w}_{\partial F}\right\|_{H^{-1 / 2}(F)}^{2} \leqslant C H_{i}{ }^{2} \omega^{2}\|\operatorname{curl} \mathbf{w}\|_{L^{2}\left(\Omega_{i}\right)}^{2} .
$$

\subsection{Decompositions associated with the faces}

After we have found the components associated with the edges, we are left with a tangential vector that vanishes along the face boundary:

$$
\mathbf{w}_{F}:=\mathbf{w}-\sum_{E \subset \partial F}\left(\mathbf{w}_{E}+\nabla_{S} \phi_{E}\right), \quad \text { on } F .
$$

Good bounds cannot be found for $\mathbf{w}_{F}$ since edge components are not logarithmically stable. We therefore consider a second face component associated with the better behaved decomposition related to $\partial F$ :

$$
\tilde{\mathbf{w}}_{F}:=\mathbf{w}-\left(\mathbf{w}_{\partial F}+\nabla_{S} \phi_{\partial F}\right), \quad \text { on } F .
$$

The following stability result is a direct consequence of Lemmas 7.9 and 7.10, and the triangle inequality. 
LEMMA 7.11 Let $F$ be a face of $\partial \Omega_{i}$. Then, for $\mathbf{w} \in X_{i}$,

$$
\begin{gathered}
\left\|\operatorname{curl}_{S} \tilde{\mathbf{w}}_{F}\right\|_{H^{-1 / 2}(F)}^{2} \leqslant C \omega^{2}\|\operatorname{curl} \mathbf{w}\|_{L_{2}\left(\Omega_{i}\right)}^{2}, \\
\left\|\tilde{\mathbf{w}}_{F}\right\|_{\mathbf{H}_{\perp}^{-1 / 2}(F)}^{2} \leqslant C \omega^{4}\left(\|\mathbf{w}\|_{L_{2}\left(\Omega_{i}\right)}^{2}+H_{i}^{2}\|\operatorname{curl} \mathbf{w}\|_{L_{2}\left(\Omega_{i}\right)}^{2}\right) .
\end{gathered}
$$

Because of the $H_{i}^{2}$ term in front of the norm of the curl, good bounds for the Maxwell discrete harmonic extension of $\tilde{\mathbf{w}}_{F}$ cannot be found directly from the previous lemma. We need to further decompose $\tilde{\mathbf{w}}_{F}$ into a curl-free and a discrete divergence-free component. The following lemma is proven in Appendix B.

LEMMA 7.12 Let $F$ be a face of $\partial \Omega_{i}$ and $\tilde{\mathbf{w}}_{F} \in W_{i}$ be a tangential vector that vanishes on all faces of $\Omega_{i}$ except on $F$. There exist $\phi_{F} \in V^{h}\left(\Omega_{i}\right)$ and $\mathbf{u}_{F} \in N D^{h}\left(\Omega_{i}\right)$, that vanish on $\partial \Omega_{i} \backslash F$, such that

$$
\tilde{\mathbf{w}}_{F}=\nabla_{S} \phi_{F}+\mathbf{u}_{F}, \quad \text { on } F
$$

and

$$
\begin{gathered}
\left\|\operatorname{curl} \mathbf{u}_{F}\right\|_{L^{2}\left(\Omega_{i}\right)}^{2} \leqslant C\left\|\operatorname{curl}_{S} \mathbf{u}_{F}\right\|_{H^{-1 / 2}(F)}^{2}=C\left\|\operatorname{curl}_{S} \tilde{\mathbf{w}}_{F}\right\|_{H^{-1 / 2}(F)}^{2}, \\
\left\|\mathbf{u}_{F}\right\|_{L^{2}\left(\Omega_{i}\right)}^{2} \leqslant C H_{i}^{2}\left\|\operatorname{curl} \mathbf{u}_{F}\right\|_{L^{2}\left(\Omega_{i}\right)}^{2}, \\
\left\|\nabla \phi_{F}\right\|_{L^{2}\left(\Omega_{i}\right)}^{2} \leqslant C\left(\left\|\tilde{\mathbf{w}}_{F}\right\|_{\mathbf{H}^{-1 / 2}(F)}^{2}+H_{i}^{2}\left\|\operatorname{curl}_{S} \tilde{\mathbf{w}}_{F}\right\|_{H^{-1 / 2}(F)}^{2}\right) .
\end{gathered}
$$

We note that no logarithmic factors appear in the first and third bounds since we have employed the stronger norms in $\mathbf{H}^{-1 / 2}(F)$ and $H^{-1 / 2}(F)$.

\subsection{Comparisons for different substructures}

In this section, we relate decompositions of functions associated with neighbouring substructures. We recall that for Algorithm A only the edge averages $a_{E}(\mathbf{w})$ are continuous and that for Algorithms B and $\mathrm{C}$ the functions $\left\langle\phi_{E}\right\rangle_{E}$ are also continuous.

We first need to relate the two decompositions (7.3) and (7.5) for the tangential component along a face boundary. The relation is given by the following lemma.

LEMMA 7.13 Let $\Omega_{i}$ be a substructure and $\mathbf{w} \in W_{i}$. Then,

$$
|\partial F| a_{\partial F}(w)=\sum_{E \subset \partial F}|E| a_{E}(w) .
$$

On every edge $E$, we have

$$
\phi_{\partial F}^{\prime}(s)=\phi_{E}^{\prime}(s)+\left(a_{E}(w)-a_{\partial F}(w)\right), \quad s \in[0,|E|],
$$

and, if, in addition, $E$ is the edge from Definition 7.3,

$$
\phi_{\partial F}(s)=\left(\phi_{E}(s)-\left\langle\phi_{E}\right\rangle_{E}\right)+\left(a_{E}(w)-a_{\partial F}(w)\right)(s-|E| / 2), \quad s \in[0,|E|] .
$$

Proof. The first and second equalities are immediate consequences of the definition of the average and of (7.3) and (7.5), respectively. The third is obtained by integrating over [0, $s]$,

$$
\phi_{\partial F}(s)=\phi_{\partial F}(0)+\phi_{E}(s)+\left(a_{E}(w)-a_{\partial F}(w)\right) s .
$$

The constant $\phi_{\partial F}(0)$ is then found by imposing that $\phi_{\partial F}(s)$ has a zero mean value along $E$. 
We have the following corollary.

Corollary 7.14 Let $F$ be a face shared by two substructures $\Omega_{i}$ and $\Omega_{j}$ and $E$ an edge of $F$. Then, for Algorithms $\mathrm{A}, \mathrm{B}$ and $\mathrm{C}$, if $\mathbf{w} \in \widetilde{W}$,

$$
a_{E}\left(\mathbf{w}^{(i)}\right)=a_{E}\left(\mathbf{w}^{(j)}\right), \quad a_{\partial F}\left(\mathbf{w}^{(i)}\right)=a_{\partial F}\left(\mathbf{w}^{(j)}\right) .
$$

We are now ready to relate the gradient contributions from different substructures.

LEMMA 7.15 Let $\Omega_{i}$ and $\Omega_{k}$ be two substructures that share a common edge $E$. Assume that $E \subset \partial F_{i}$ and $E \subset \partial F_{k}$, with $F_{i}$ and $F_{k}$ faces of $\Omega_{i}$ and $\Omega_{k}$, respectively, that do not necessarily coincide. Let $\mathbf{w} \in \widetilde{W}$, with local components $\mathbf{w}^{(i)} \in W_{i}$ and $\mathbf{w}^{(k)} \in W_{k}$. If the edge $E$ is that from Definition 7.3, then for Algorithms A, B and C and $s \in[0,|E|]$,

$$
\phi_{E}^{(i)}-\phi_{E}^{(k)}=\left(\phi_{\partial F_{i}}^{(i)}-\phi_{\partial F_{k}}^{(k)}\right)+\left(\left\langle\phi_{E}^{(i)}\right\rangle_{E}-\left\langle\phi_{E}^{(k)}\right\rangle_{E}\right)+\left(a_{\partial F_{i}}\left(w^{(i)}\right)-a_{\partial F_{k}}\left(w^{(k)}\right)\right)(s-|E| / 2) .
$$

In particular, if the two substructures share the face $F=F_{i}=F_{k}$, then

$$
\phi_{E}^{(i)}-\phi_{E}^{(k)}=\left(\phi_{\partial F}^{(i)}-\phi_{\partial F}^{(k)}\right)+\left(\left\langle\phi_{E}^{(i)}\right\rangle_{E}-\left\langle\phi_{E}^{(k)}\right\rangle_{E}\right) .
$$

Proof. The result is a direct consequence of Lemma 7.13 and Corollary 7.14.

We also have a comparison result for face components. It employs the continuous, piecewise trilinear function $\theta_{\partial F}$ that is identically one on $\partial F$ and vanishes at all the nodes inside $F$.

LEMMA 7.16 Let $\Omega_{i}$ and $\Omega_{j}$ be two substructures that share a common face $F$. Let, in addition, w $\in \widetilde{W}$, with local components $\mathbf{w}^{(i)} \in W_{i}$ and $\mathbf{w}^{(k)} \in W_{k}$. For Algorithms A, B and C, we have, on $F$,

$$
\mathbf{w}_{F}^{(i)}-\mathbf{w}_{F}^{(j)}=\tilde{\mathbf{w}}_{F}^{(i)}-\tilde{\mathbf{w}}_{F}^{(j)}-\left(\left\langle\phi_{E}^{(i)}\right\rangle_{E}-\left\langle\phi_{E}^{(j)}\right\rangle_{E}\right) \nabla_{S} \theta_{\partial F},
$$

with $E$ the edge from Definition 7.3.

Proof. The proof relies on (7.6) and (7.7). We have,

$$
\begin{gathered}
\mathbf{w}_{F}^{(i)}-\mathbf{w}_{F}^{(j)}=\left(\tilde{\mathbf{w}}_{F}^{(i)}-\tilde{\mathbf{w}}_{F}^{(j)}\right)+\left(\mathbf{w}_{\partial F}^{(i)}-\mathbf{w}_{\partial F}^{(j)}\right)-\sum_{E \subset \partial F}\left(\mathbf{w}_{E}^{(i)}-\mathbf{w}_{E}^{(j)}\right) \\
-\sum_{E \subset \partial F}\left(\nabla_{S} \phi_{E}^{(i)}-\nabla_{S} \phi_{E}^{(j)}\right)+\left(\nabla_{S} \phi_{\partial F}^{(i)}-\nabla_{S} \phi_{\partial F}^{(j)}\right) .
\end{gathered}
$$

Corollary 7.14 implies that the second and third terms on the right-hand side vanish and thus

$$
\begin{gathered}
\mathbf{w}_{F}^{(i)}-\mathbf{w}_{F}^{(j)}=\left(\tilde{\mathbf{w}}_{F}^{(i)}-\tilde{\mathbf{w}}_{F}^{(j)}\right)+\nabla_{S} r \\
r=\left(\phi_{\partial F}^{(i)}-\sum_{E \subseteq \partial F} \phi_{E}^{(i)}\right)-\left(\phi_{\partial F}^{(j)}-\sum_{E \subset \partial F} \phi_{E}^{(j)}\right) .
\end{gathered}
$$

We now take a closer look at the remainder $\nabla_{S} r$. We first note that $r$ vanishes at all the nodes inside $F$. We next consider the tangential component along $\partial F$. Using Lemma 7.13, we find, along $\partial F$,

$$
\nabla_{S} r \cdot \mathbf{t}_{\partial F}=\sum_{E \subset \partial F}\left(a_{E}\left(w^{(i)}\right)-a_{E}\left(w^{(j)}\right)\right) \Phi_{E}-\left(a_{\partial F}\left(w^{(i)}\right)-a_{\partial F}\left(w^{(j)}\right)\right),
$$


with $\Phi_{E}$ the characteristic function of $E$. Corollary 7.14 implies that the right-hand side is zero and $r$ is therefore constant on $\partial F$. In order to find this constant value, we find the mean value $\langle r\rangle_{E}$ with $E$ the edge from Definition 7.3. We have

$$
r=\langle r\rangle_{E}=-\left(\left\langle\phi_{E}^{(i)}\right\rangle_{E}-\left\langle\phi_{E}^{(j)}\right\rangle_{E}\right), \quad \text { on } F,
$$

which concludes the proof.

We stress that the differences between components over different substructures depend on the averages $\left\langle\phi_{E}\right\rangle_{E}$, which are continuous for Algorithms B and C, or the averages $a_{\partial F}(w)$ for which good bounds have been found in Lemma 7.5.

\section{Main result}

The FETI operator and the preconditioner are invertible for the three algorithms considered. The proof is the same as that of Toselli \& Vasseur (2004, Lemma 4.5) and is a direct consequence of the invertibility of the local bilinear forms on the subdomains.

LEMMA 8.1 The Schur complement $\widetilde{S}$ and the preconditioner $M^{-1}$ for Algorithms A, B and C are invertible.

We are now ready to prove our main result, which is a logarithmic bound for the condition numbers of Algorithms B and C, independent of the coefficient jumps; see Theorem 4.2.

Lemma 8.2 (Algorithms B And C) For Algorithms B and C, there is a constant, independent of $h$, $H$ and the coefficients $A$ and $B$, such that, for $\mathbf{w}_{\Delta} \in \widetilde{W}_{\Delta}$,

$$
\left|P_{\Delta} \mathbf{w}_{\Delta}\right|_{S}^{2} \leqslant C \eta(1+\log (H / h))^{4}\left|\mathbf{w}_{\Delta}\right|_{\widetilde{S}}^{2}
$$

where

$$
\eta:= \begin{cases}\max _{1 \leqslant i \leqslant N} \max \left\{\frac{\gamma_{i}}{\beta_{i}}, 1+\frac{H_{i}^{2} \gamma_{i}}{a}\right\} & \text { for Case } 1, \\ \max _{1 \leqslant i \leqslant N} \max \left\{\frac{\gamma_{i}}{\beta_{i}}, 1+\frac{H_{i}^{2} b}{\beta_{i}}\right\} & \text { for Case } 2 .\end{cases}
$$

Therefore, the condition number of the corresponding preconditioned FETI operators satisfies

$$
\kappa\left(M^{-1} F\right) \leqslant C \eta(1+\log (H / h))^{4} .
$$

Proof. Here, we only consider the coefficient distribution of Case 1 in full detail; see (2.1). Case 2 can be dealt with in the same way. Using the minimization property in (4.3), we consider the element $\mathbf{w}=\mathbf{w}_{\Delta}+\mathbf{w}_{\Pi}, \mathbf{w}_{\Pi} \in \widehat{W}_{\Pi}$ such that

$$
\left|\mathbf{w}_{\Delta}\right|_{\widetilde{S}}^{2}=|\mathbf{w}|_{S}^{2}=\sum_{i=1}^{N}\left|\mathbf{w}^{(i)}\right|_{S^{(i)}}^{2}=\sum_{i=1}^{N} a_{\Omega_{i}}\left(\mathcal{H}_{i} \mathbf{w}^{(i)}, \mathcal{H}_{i} \mathbf{w}^{(i)}\right),
$$

where for the last inequality we have used the minimization property of the discrete harmonic extension. We note that, since $\mathbf{w}_{\Pi}$ is continuous, Lemma 4.1 ensures that

$$
\mathbf{v}:=P_{\Delta} \mathbf{w}_{\Delta}=P_{\Delta} \mathbf{w} .
$$


We then need to calculate

$$
\left|P_{\Delta} \mathbf{w}\right|_{S}^{2}=\sum_{i=1}^{N}\left|\mathbf{v}^{(i)}\right|_{S^{(i)}}^{2}=\sum_{i=1}^{N} a_{\Omega_{i}}\left(\mathcal{H}_{i} \mathbf{v}^{(i)}, \mathcal{H}_{i} \mathbf{v}^{(i)}\right) .
$$

On each subdomain $\Omega_{i}$, the tangential vector $\mathbf{v}^{(i)}$ is given by formula (4.9). We recall that this formula is to be understood for vectors of degrees of freedom, which are those in the new basis of Definition 6.1. These are the averages along the subdomain edges, the nodal values that determine the gradient components along the edges and the tangential components along fine edges that lie in the interior of the faces, and they determine the decomposition (6.1) into edge and face components. In addition, the vector of degrees of freedom $\delta_{j}^{\dagger}$ takes the same value $\delta_{j}^{\dagger}(F)$ on a subdomain face $F$ and the same value $\delta_{j}^{\dagger}(E)$ on a subdomain edge $E$. It is therefore natural to decompose $\mathbf{v}^{(i)}$ into terms associated with single edges and faces; see (6.1). We obtain

$$
\begin{aligned}
\mathbf{v}^{(i)}= & \sum_{E} \mathbf{v}_{E}+\sum_{E} \nabla_{S} \phi_{E}+\sum_{F_{i j}} \mathbf{v}_{F_{i j}} \\
= & \sum_{E} \sum_{k} \delta_{k}^{\dagger}(E)\left(\mathbf{w}_{E}^{(i)}-\mathbf{w}_{E}^{(k)}\right)+\sum_{E} \sum_{k} \delta_{k}^{\dagger}(E) \nabla_{S}\left(\phi_{E}^{(i)}-\phi_{E}^{(k)}\right) \\
& +\sum_{F_{i j}} \delta_{j}^{\dagger}\left(F_{i j}\right)\left(\mathbf{w}_{F_{i j}}^{(i)}-\mathbf{w}_{F_{i j}}^{(j)}\right),
\end{aligned}
$$

where a face $F_{i j}$ is assumed to be shared by $\Omega_{i}$ and $\Omega_{j}$ and the sums over $k$ are taken over the subdomains $\Omega_{k}$ that share the edge $E$. Since for functions in $\widetilde{W}$ averages of the tangential component along an edge $E$ are all equal for all the algorithms considered, the constant edge contributions $\mathbf{w}_{E}^{(i)}$ and $\mathbf{w}_{E}^{(j)}$ are equal and the corresponding sums on the right-hand side of (8.2) vanish; cf. Corollary 7.14. We are therefore left with two terms, which we consider separately.

Edge terms. We first note that for Algorithm C, these terms vanish since all degrees of freedom are continuous across a coarse edge in this case. For Algorithm B, we consider an edge $E$ and two subdomains $\Omega_{i}$ and $\Omega_{k}$ that share the edge $E$.

We first assume that the two substructures also share a whole face $F$. We consider the term

$$
\delta_{k}^{\dagger}(E) \nabla_{S}\left(\phi_{E}^{(i)}-\phi_{E}^{(k)}\right)
$$

It involves a tangential vector on the boundary of $\Omega_{i}$ and in order to evaluate its $|\cdot|_{S^{(i)}}$ norm, we need to evaluate the energy of its discrete Maxwell extension into $\Omega_{i}$. Using the minimizing property of the Maxwell extension and Lemma 7.4, we find

$$
\left|\delta_{k}^{\dagger}(E) \nabla_{S}\left(\phi_{E}^{(i)}-\phi_{E}^{(k)}\right)\right|_{S^{(i)}}^{2} \leqslant C \gamma_{i} \delta_{k}^{\dagger}(E)^{2}\left\|\phi_{E}^{(i)}-\phi_{E}^{(k)}\right\|_{L^{2}(E)}^{2} .
$$

We assume that we have chosen $E$ as the edge from Definition 7.3. Using Lemma 7.15 with $F=F_{i}=$ $F_{k}$, and the fact that $\left\langle\phi_{E}^{(i)}\right\rangle_{E}=\left\langle\phi_{E}^{(k)}\right\rangle_{E}$, we can write along $E$,

$$
\phi_{E}^{(i)}-\phi_{E}^{(k)}=\phi_{\partial F}^{(i)}-\phi_{\partial F}^{(k)},
$$

and, thus

$$
\begin{aligned}
\left|\delta_{k}^{\dagger}(E) \nabla_{S}\left(\phi_{E}^{(i)}-\phi_{E}^{(k)}\right)\right|_{S^{(i)}}^{2} & \leqslant C \gamma_{i} \delta_{k}^{\dagger}(E)^{2}\left\|\phi_{\partial F}^{(i)}-\phi_{\partial F}^{(k)}\right\|_{L^{2}(E)}^{2} \\
& \leqslant C \gamma_{i} \delta_{k}^{\dagger}(E)^{2}\left(\left\|\phi_{\partial F}^{(i)}\right\|_{L^{2}(\partial F)}^{2}+\left\|\phi_{\partial F}^{(k)}\right\|_{L^{2}(\partial F)}^{2}\right) .
\end{aligned}
$$


The last two terms can then be bounded using Lemma 7.8 and inequality (4.7). For the first, we find

$$
\gamma_{i} \delta_{k}^{\dagger}(E)^{2}\left\|\phi_{\partial F}^{(i)}\right\|_{L^{2}(\partial F)}^{2} \leqslant C \gamma_{i} \omega^{2}\left(\left\|\mathbf{w}^{(i)}\right\|_{L^{2}\left(\Omega_{i}\right)}^{2}+H_{i}^{2}\left\|\mathbf{c u r l} \mathbf{w}^{(i)}\right\|_{L^{2}\left(\Omega_{i}\right)}^{2}\right) \leqslant C \eta \omega^{2}\left|\mathbf{w}^{(i)}\right|_{S^{(i)}}^{2},
$$

where we have used the same notation for $\mathbf{w}^{(i)} \in W_{i}$ and the corresponding Maxwell extension in $\Omega_{i}$. A similar reasoning on $\Omega_{k}$ gives a bound for the second term. We have therefore found

$$
\left|\delta_{k}^{\dagger}(E) \nabla_{S}\left(\phi_{E}^{(i)}-\phi_{E}^{(k)}\right)\right|_{S^{(i)}}^{2} \leqslant C \eta(1+\log (H / h))^{2}\left(\left|\mathbf{w}^{(i)}\right|_{S^{(i)}}^{2}+\left|\mathbf{w}^{(i)}\right|_{S^{(k)}}^{2}\right) .
$$

We now consider the case of two substructures $\Omega_{i}$ and $\Omega_{k}$ that share an edge $E$ but not a full face. We note that in this case, (8.3) does not hold. Lemma 7.15 gives

$$
\phi_{E}^{(i)}-\phi_{E}^{(k)}=\phi_{\partial F_{i}}^{(i)}-\phi_{\partial F_{k}}^{(k)}+\left(a_{\partial F_{i}}\left(w^{(i)}\right)-a_{\partial F_{k}}\left(w^{(k)}\right)\right)(s-|E| / 2) .
$$

The first two terms on the right-hand side can be bounded in the same way as before. We are left with two terms involving the face averages. Using Lemma 7.5 yields

$$
\begin{aligned}
& \gamma_{i} \delta_{k}^{\dagger}(E)^{2}\left\|a_{\partial F_{i}}\left(w^{(i)}\right)(s-|E| / 2)\right\|_{L^{2}(E)}^{2} \\
& \quad \leqslant C \gamma_{i}\left|a_{\partial F_{i}}\left(w^{(i)}\right)\right|^{2}\|s-|E| / 2\|_{L^{2}(E)}^{2} \\
& \quad \leqslant C \gamma_{i} \omega^{2} H_{i}^{2} \| \mathbf{c u r l} \mathbf{w}\left({ }^{(i)} \|_{L^{2}\left(\Omega_{i}\right)}^{2} \leqslant C \eta \omega^{2}\left|\mathbf{w}^{(i)}\right|_{S^{(i)}}^{2} .\right.
\end{aligned}
$$

An analogous bound holds for the term corresponding to $\Omega_{k}$ and, therefore, (8.4) also holds in this case.

Face terms. We now consider the sum involving face terms on the right-hand side of (8.2). We fix a face $F=F_{i j}$. Lemma 7.16 ensures

$$
\mathbf{w}_{F}^{(i)}-\mathbf{w}_{F}^{(j)}=\tilde{\mathbf{w}}_{F}^{(i)}-\tilde{\mathbf{w}}_{F}^{(j)} .
$$

We employ the decomposition of Lemma 7.12 and find

$$
\delta_{j}^{\dagger}(F)\left(\mathbf{w}_{F}^{(i)}-\mathbf{w}_{F}^{(j)}\right)=\delta_{j}^{\dagger}(F)\left(\nabla_{S} \phi_{F}^{(i)}-\nabla_{S} \phi_{F}^{(j)}\right)+\delta_{j}^{\dagger}(F)\left(\mathbf{u}_{F}^{(i)}-\mathbf{u}_{F}^{(j)}\right) .
$$

For the gradient term $\nabla_{S} \phi_{F}^{(i)}$, Lemmas 7.12 and 7.11 ensure

$$
\begin{aligned}
\left|\delta_{j}^{\dagger}(F) \nabla_{S} \phi_{F}^{(i)}\right|_{S^{(i)}}^{2} & \leqslant \delta_{j}^{\dagger}(F)^{2} a_{\Omega_{i}}\left(\nabla \phi_{F}^{(i)}, \nabla \phi_{F}^{(i)}\right) \leqslant \gamma_{i} \delta_{j}^{\dagger}(F)^{2}\left\|\nabla \phi_{F}^{(i)}\right\|_{L^{2}\left(\Omega_{i}\right)}^{2} \\
& \leqslant C \gamma_{i}\left(\left\|\tilde{\mathbf{w}}_{F}^{(i)}\right\|_{\mathbf{H}_{\perp}^{-1 / 2}(F)}^{2}+H_{i}^{2}\left\|\operatorname{curl}_{S} \tilde{\mathbf{w}}_{F}^{(i)}\right\|_{H^{-1 / 2}(F)}^{2}\right) \\
& \leqslant C \gamma_{i} \omega^{4}\left(\left\|\mathbf{w}^{(i)}\right\|_{L_{2}\left(\Omega_{i}\right)}^{2}+H_{i}^{2}\left\|\operatorname{curl} \mathbf{w}^{(i)}\right\|_{L_{2}\left(\Omega_{i}\right)}^{2}\right) \\
& \leqslant C \eta(1+\log (H / h))^{4}\left|\mathbf{w}^{(i)}\right|_{S^{(i)}}^{2} .
\end{aligned}
$$

The term involving $\nabla_{S} \phi_{F}^{(j)}$ can be bounded in a similar way. We, therefore, find

$$
\left|\delta_{j}^{\dagger}(F)\left(\nabla_{S} \phi_{F}^{(i)}-\nabla_{S} \phi_{F}^{(j)}\right)\right|_{S^{(i)}}^{2} \leqslant C \eta(1+\log (H / h))^{4}\left(\left|\mathbf{w}^{(i)}\right|_{S^{(i)}}^{2}+\left|\mathbf{w}^{(j)}\right|_{S^{(j)}}^{2}\right) .
$$


The second term in (8.5) is associated with the curl operator and is the sum of two contributions, which we consider separately. For the first, Lemmas 7.12 and 7.11 give

$$
\begin{aligned}
\left|\delta_{j}^{\dagger}(F) \mathbf{u}_{F}^{(i)}\right|_{S^{(i)}}^{2} & \leqslant \delta_{j}^{\dagger}(F)^{2} a_{\Omega_{i}}\left(\mathbf{u}_{F}^{(i)}, \mathbf{u}_{F}^{(i)}\right) \leqslant \delta_{j}^{\dagger}(F)^{2}\left(a+\gamma_{i} H_{i}^{2}\right)\left\|\operatorname{curl} \mathbf{u}_{F}^{(i)}\right\|_{L^{2}\left(\Omega_{i}\right)}^{2} \\
& \leqslant \delta_{j}^{\dagger}(F)^{2}\left(a+\gamma_{i} H_{i}^{2}\right)\left\|\operatorname{curl}_{S} \tilde{\mathbf{w}}_{F}^{(i)}\right\|_{H^{-1 / 2}(F)}^{2} \\
& \leqslant C \delta_{j}^{\dagger}(F)^{2} \omega^{2}\left(a+\gamma_{i} H_{i}^{2}\right)\left\|\operatorname{curl} \mathbf{w}^{(i)}\right\|_{L^{2}\left(\Omega_{i}\right)}^{2} \\
& \leqslant C \omega^{2}\left(1+\left(\gamma_{i} H_{i}^{2}\right) / a\right)\left\|a^{1 / 2} \operatorname{curl} \mathbf{w}^{(i)}\right\|_{L^{2}\left(\Omega_{i}\right)}^{2} \\
& \leqslant C \omega^{2} \eta\left\|a^{1 / 2} \operatorname{curl} \mathbf{w}^{(i)}\right\|_{L^{2}\left(\Omega_{i}\right)}^{2} \\
& \leqslant C \omega^{2} \eta\left(\left\|B^{1 / 2} \mathbf{w}^{(i)}\right\|_{L^{2}\left(\Omega_{i}\right)}^{2}+\left\|a^{1 / 2} \operatorname{curl~w}^{(i)}\right\|_{L^{2}\left(\Omega_{i}\right)}^{2}\right) \\
& =C(1+\log (H / h))^{2} \eta\left\|\mathbf{w}^{(i)}\right\|_{S^{(i)}}^{2} .
\end{aligned}
$$

For the term $\mathbf{u}_{F}^{(j)}$ in (8.5), we can reason in a similar way. Using (8.6), we obtain the bound

$$
\left|\delta_{j}^{\dagger}(F)\left(\mathbf{w}_{F}^{(i)}-\mathbf{w}_{F}^{(j)}\right)\right|_{S^{(i)}}^{2} \leqslant C \eta(1+\log (H / h))^{4}\left(\left|\mathbf{w}^{(i)}\right|_{S^{(i)}}^{2}+\left|\mathbf{w}^{(j)}\right|_{S^{(j)}}^{2}\right) .
$$

The proof is concluded by combining (8.2), (8.4) and (8.7) and summing over the substructures $\Omega_{i}$.

We note that the bound in the previous lemma is not likely to be sharp. Indeed, the results in Tables 2 and 3 are consistent with a quadratic growth with $\log (H / h)$, which is typical of many iterative substructuring algorithms.

TABLE 1 Algorithm A. Estimated condition number and number of CG iterations (in parentheses) versus $H / h$ and $n$. Case of constant, unit coefficients $A$ and $B$

\begin{tabular}{lccccc}
\hline$H / h$ & 8 & 6 & 4 & 3 & 2 \\
\hline$n=8$ & - & - & $7.085(15)$ & - & $3.134(18)$ \\
$n=16$ & $19.38(20)$ & - & $8.962(33)$ & - & $3.179(19)$ \\
$n=24$ & $25.72(37)$ & $17.39(43)$ & $9.306(35)$ & $5.879(27)$ & $3.184(18)$ \\
$n=32$ & $27.45(46)$ & - & $9.382(35)$ & - & $3.186(17)$ \\
$n=40$ & $28.01(57)$ & - & $9.449(35)$ & - & - \\
$n=48$ & $28.24(59)$ & $18.12(48)$ & $9.487(34)$ & $5.94(26)$ & - \\
\hline
\end{tabular}

TABLE 2 Algorithm B. Estimated condition number and number of $C G$ iterations (in parentheses) versus $H / h$ and $n$. Case of constant, unit coefficients $A$ and $B$

\begin{tabular}{lccccc}
\hline$H / h$ & 8 & 6 & 4 & 3 & 2 \\
\hline$n=8$ & - & - & $2.213(12)$ & - & $1.869(13)$ \\
$n=16$ & $3.076(15)$ & - & $2.742(18)$ & - & $1.936(13)$ \\
$n=24$ & $3.566(20)$ & $3.322(20)$ & $2.838(18)$ & $2.48(16)$ & $1.960(13)$ \\
$n=32$ & $3.774(22)$ & - & $2.899(18)$ & - & $1.969(13)$ \\
$n=40$ & $3.866(22)$ & - & $2.926(18)$ & - & - \\
$n=48$ & $3.951(22)$ & $3.537(20)$ & $2.944(18)$ & - & - \\
\hline
\end{tabular}


TABLE 3 Algorithm C. Estimated condition number and number of CG iterations (in parentheses) versus $H / h$ and $n$. Case of constant, unit coefficients $A$ and $B$

\begin{tabular}{lccccc}
\hline$H / h$ & 8 & 6 & 4 & 3 & 2 \\
\hline$n=8$ & - & - & $2.211(12)$ & - & $1.869(13)$ \\
$n=16$ & $3.068(16)$ & - & $2.741(17)$ & - & $1.936(13)$ \\
$n=24$ & $3.558(20)$ & $3.318(20)$ & $2.837(18)$ & $2.479(16)$ & $1.960(13)$ \\
$n=32$ & $3.766(21)$ & - & $2.899(18)$ & - & $1.969(13)$ \\
$n=40$ & $3.859(22)$ & - & $2.929(18)$ & - & - \\
$n=48$ & $3.945(22)$ & $3.536(20)$ & - & - & - \\
\hline
\end{tabular}

REMARK 8.3 For Algorithm A, we expect the bound

$$
\kappa\left(M^{-1} F\right) \leqslant C \eta(H / h)(1+\log (H / h))^{q} .
$$

An analysis similar to that discussed above would provide a bound with $q=4$, while the results in Table 1 are consistent with $q=2$. More precisely, when bounding the edge terms associated with the decomposition (8.2), additional terms involving the averages $\left\langle\phi_{E}^{(i)}\right\rangle_{E}$ and $\left\langle\phi_{E}^{(k)}\right\rangle_{E}$ need to be considered; see Lemma 7.15. Logarithmic bounds for these averages cannot be found. Analogous considerations apply to the face contributions; see Lemma 7.16. Alternatively, we could simply ask that the functions $\phi_{\partial F}$ in Definition 7.3 only vanish at one vertex for Algorithm A: this would simplify Lemmas 7.15 and 7.16 but good bounds cannot be found in Lemma 7.8 for a function that only vanishes at a point of $\partial F$; see Remark 7.7.

\section{Numerical results}

In this section, we present some numerical results on the performance of the three algorithms proposed in this paper.

We consider the domain $\Omega=(0,1)^{3}$ and uniform triangulations $\mathcal{T}_{h}$ and $\mathcal{T}_{H}$. The coarse triangulation $\mathcal{T}_{H}$ consists of $N^{3}$ cubical elements, with $H=1 / N$. The fine one $\mathcal{T}_{h}$ is a refinement of $\mathcal{T}_{H}$ and consists of $n^{3}$ cubical elements, with $h=1 / n$. The substructures $\Omega_{i}$ are chosen as the elements of $\mathcal{T}_{H}$. The matrices $A$ and $B$ are always multiples of the identity; cf. (2.1). We employ the value $\chi=1 / 2$ for the definition of the scaling matrices $D_{\Delta}^{(i)}$; see (4.6). We consider a CG algorithm and estimate the condition number of the preconditioned operator using the quantities provided by CG. We stop the iteration when $\left\|z_{k}\right\| /\|\mathbf{f}\|$ is less than $10^{-12}$, where $z_{k}$ is the $k$-th preconditioned residual $M^{-1}\left(d-F \lambda_{k}\right)$.

The purpose of these simple tests is to assess the scalability, quasi-optimality and robustness with respect to coefficient jumps of our algorithms. In addition, they show that low condition numbers and iteration counts typical of FETI methods are also found for edge-element approximations and that the bounds that we derived are not sharp, neither in terms of $H / h$ nor in terms of the parameter $\eta$.

We first consider the case where both coefficients are identical. Tables 1, 2 and 3 show the estimated condition number and the number of iterations, as a function of the dimensions of the fine mesh and $H / h=n / N$, for Algorithms A, B and C, respectively. For a fixed $H / h$, the condition number appears to remain bounded, independent of the number of fine mesh points $n$. As expected, for a fixed ratio $H / h$, the condition number and the number of iterations are quite insensitive to the dimension of the fine mesh. However, considerably higher iteration counts and condition numbers are found for Algorithm A; 
Algorithms B and C show the typically low condition numbers of FETI methods; see, e.g. Toselli \& Klawonn (2001) and Toselli \& Vasseur (2004) for 2D results on edge elements. See also Toselli \& Widlund (2004, Chapter 6), where the results originally presented in Klawonn et al. (2004) are reported. We note that, surprisingly, the much larger coarse space of Algorithm C does not translate into a much smaller number of iterations or condition number. On the other hand, results for the two algorithms are comparable (for the case $H / h=2$, they provide the same results). Some cases with very small values of $H / h$ give rise to very large coarse problems for Algorithm $\mathrm{C}$ and cannot be run. The interest for Algorithm $\mathrm{C}$ is that it does not require a change of basis and can be potentially attractive for $p$ and $h p$ edge-element approximations where the required change of basis is less local.

The results for Algorithms B and C are consistent with a quadratic growth with $\log (H / h)$, hinting that the result in Lemma 8.2 is not sharp. For Algorithm A, we refer to Remark 8.3.

In order to show the necessity of a change of basis, we report in Table 4 the condition numbers of Algorithm B employed without performing a change of basis, for the same cases as in Table 2 . The condition numbers become very high and are consistent with a quadratic growth in $n=1 / h$, thus confirming our analysis in Section 5. For $H / h=2$, there are only two degrees of freedom along a subdomain edge

TABLE 4 Algorithm B without change of basis. Condition number versus $H / h$ and $n$. Case of constant, unit coefficients $A$ and $B$

\begin{tabular}{lccccc}
\hline$H / h$ & 8 & 6 & 4 & 3 & 2 \\
\hline$n=8$ & - & - & 151.6 & - & 1.869 \\
$n=16$ & 643.7 & - & 607.6 & - & 1.936 \\
$n=24$ & 1449 & 1429 & 1365 & 1429 & 1.960 \\
$n=32$ & 2576 & - & 2427 & - & 1.969 \\
$n=40$ & 4024 & - & 3793 & - & - \\
$n=48$ & 5795 & 5712 & 5462 & - & - \\
\hline
\end{tabular}

TABLE 5 Algorithm B. $A 4 \times 4 \times 4$ checkerboard distribution for $b:\left(b_{1}, b_{2}\right)$. Estimated condition number and number of CG iterations (in parentheses) versus $H / h$ and $b_{2}$. Case of $n=32, a=1$ and $b_{1}=100$

\begin{tabular}{lccc}
\hline$H / h$ & 8 & 4 & 2 \\
\hline$b_{2}=1 \times 10^{-4}$ & $10.47(58)$ & $7.283(46)$ & $4.588(34)$ \\
$b_{2}=1 \times 10^{-3}$ & $10.46(56)$ & $7.282(46)$ & $4.587(32)$ \\
$b_{2}=1 \times 10^{-2}$ & $10.45(56)$ & $7.278(44)$ & $4.586(32)$ \\
$b_{2}=1 \times 10^{-1}$ & $10.37(52)$ & $7.252(42)$ & $4.572(30)$ \\
$b_{2}=1$ & $9.728(40)$ & $7.075(33)$ & $4.477(25)$ \\
$b_{2}=1 \times 10^{1}$ & $7.219(33)$ & $5.696(29)$ & $3.739(22)$ \\
$b_{2}=1 \times 10^{2}$ & $2.549(16)$ & $2.254(14)$ & $1.739(11)$ \\
$b_{2}=1 \times 10^{3}$ & $6.023(30)$ & $3.968(23)$ & $3.267(20)$ \\
$b_{2}=1 \times 10^{4}$ & $8.747(36)$ & $3.902(24)$ & $2.935(19)$ \\
$b_{2}=1 \times 10^{5}$ & $11.25(52)$ & $3.819(32)$ & $1.977(20)$ \\
$b_{2}=1 \times 10^{6}$ & $11.97(52)$ & $3.836(34)$ & $1.746(18)$ \\
\hline
\end{tabular}


TABLE 6 Algorithm B. $A 4 \times 4 \times 4$ checkerboard distribution for $a:\left(a_{1}, a_{2}\right)$. Estimated condition number and number of CG iterations (in parentheses) versus $H / h$ and $a_{2}$. Case of $n=32, b=1$ and $a_{1}=0.01$

\begin{tabular}{lccc}
\hline$H / h$ & 8 & 4 & 2 \\
\hline$a_{2}=1 \times 10^{-7}$ & $8.152(30)$ & $3.722(20)$ & $1.896(12)$ \\
$a_{2}=1 \times 10^{-6}$ & $8.102(30)$ & $3.704(20)$ & $1.901(12)$ \\
$a_{2}=1 \times 10^{-5}$ & $7.687(29)$ & $3.554(19)$ & $1.937(12)$ \\
$a_{2}=1 \times 10^{-4}$ & $6.282(27)$ & $3.061(18)$ & $2.229(14)$ \\
$a_{2}=1 \times 10^{-3}$ & $4.707(23)$ & $3.015(17)$ & $2.255(14)$ \\
$a_{2}=1 \times 10^{-2}$ & $2.549(16)$ & $2.254(14)$ & $1.739(11)$ \\
$a_{2}=1 \times 10^{-1}$ & $4.674(24)$ & $3.238(19)$ & $2.289(15)$ \\
$a_{2}=1$ & $5.450(27)$ & $3.782(21)$ & $2.680(17)$ \\
$a_{2}=1 \times 10^{1}$ & $5.545(27)$ & $3.851(22)$ & $2.702(22)$ \\
$a_{2}=1 \times 10^{2}$ & $5.484(32)$ & $3.801(28)$ & $2.707(22)$ \\
$a_{2}=1 \times 10^{3}$ & $5.486(32)$ & $3.802(28)$ & $2.707(22)$ \\
\hline
\end{tabular}

and the two primal constraints therefore enforce the continuity of the edge-element vectors along an edge. We remark that adding more primal constraints, such as face averages or higher-order moments along the edges, does not remove this dependence on $h$ (the results are not presented here).

Despite the fact that our condition number bound blows up when the coefficient $A$ goes to zero, the algorithms remain robust in practice (results are not shown here), in exactly the same way as for many iterative substructuring algorithms for Raviart-Thomas and 2D edge-element approximations; see Toselli et al. (2001), Wohlmuth (2000), Toselli (2000a) and Toselli \& Klawonn (2001).

We now focus our attention on Algorithm B and problems with coefficient jumps. We first consider jumps in $B$; see Case 1 in (2.1). We choose a $4 \times 4 \times 4$ checkerboard distribution, where $B$ assumes two values, $b_{1}$ and $b_{2}$. For a fixed value of $n=32, b_{1}=100$ and $a=1$, Table 5 shows the estimated condition number and the number of iterations, as a function of $H / h$ and $b_{2}$. The algorithm appears to be robust with respect to large coefficient jumps. The results for a case of jumps in the coefficient $A$, see Case 2 in (2.1), are shown in Table 6. We choose a $4 \times 4 \times 4$ checkerboard distribution, where $A$ assumes two values, $a_{1}=0.01$ and $a_{2}$. The same conclusions as for Table 5 can be drawn in this case. Algorithms A and $\mathrm{C}$ show an analogous behaviour (the results are not presented here). We note that for Cases 1 and 2, iteration counts and condition numbers remain bounded when the coefficient $A$ goes to zero (or $B$ is large) on some subdomains; see $\eta$ in Lemma 8.2. The case when jumps are present in both coefficients remains open.

\section{Acknowledgements}

The author would like to express his gratitude to A. Buffa who gave him precious insight into available results on traces of vector spaces and on some key parts of the analysis in Section 7. This work greatly benefited from some early discussions with B. Wohlmuth and O. Widlund and, more recently, with Ch. Schwab. This work was partially supported by the Swiss National Science Foundation under Project 20-63397.00. 


\section{REFERENCES}

Alonso, A. \& VAlli, A. (1996) Some remarks on the characterization of the space of tangential traces of $H(\operatorname{rot} ; \omega)$ and the construction of an extension operator. Manuscripta Math., 89, 159-178.

Amrouche, C., Bernardi, C., Dauge, M. \& Girault, V. (1998) Vector potentials in three-dimensional non-smooth domains. Math. Methods Appl. Sci., 21, 823-864.

Bhardwaj, M., Pierson, K., Reese, G., Walsh, T., Day, D., Alvin, K., Peery, J., Farhat, C. \& LESoinne, M. (2002) Salinas: a scalable software for high-performance structural and solid mechanics simulations. Proceedings of the IEEE/ACM SC2002 Conference, Baltimore, MD, 16-22 November 2002. Los Alamos: IEEE Computer Society Press.

Brezzi, F. \& Fortin, M. (1991) Mixed and Hybrid Finite Element Methods. New York: Springer.

Buffa, A. \& CiARlet JR., P. (2001a) On traces for functional spaces related to Maxwell's equations. I. An integration by parts formula in Lipschitz polyhedra. Math. Methods Appl. Sci., 24, 9-30.

Buffa, A. \& Ciarlet JR., P. (2001b) On traces for functional spaces related to Maxwell's equations. II. Hodge decompositions on the boundary of Lipschitz polyhedra and applications. Math. Methods Appl. Sci., 24, $31-48$.

Butrylo, B., Musy, F., Nicolas, L., Perrussel, R., Scorretti, R. \& Vollaire, C. (2004) A survey of parallel solvers for the finite element method in computational electromagnetics. COMPEL, 23, 531-546.

Dahmen, W., Faermann, B., Graham, I. G., Hackbusch, W. \& Sauter, S. A. (2004) Inverse inequalities on non-quasi-uniform meshes and application to the mortar element method. Math. Comput., 73, 1107-1138.

Dryja, M., Smith, B. F. \& WidLund, O. B. (1994) Schwarz analysis of iterative substructuring algorithms for elliptic problems in three dimensions. SIAM J. Numer. Anal., 31, 1662-1694.

Farhat, C., Lesoinne, M., LeTallec, P., Pierson, K. \& Rixen, D. (2001) FETI-DP: a dual-primal unified FETI method. I. A faster alternative to the two-level FETI method. Int. J. Numer. Meth. Eng., 50, 1523-1544.

FArhat, C., Lesoinne, M. \& Pierson, K. (2000) A scalable dual-primal domain decomposition method. Numer. Linear Algebra Appl., 7, 687-714.

FARHAT, C. \& RouX, F.-X. (1994) Implicit parallel processing in structural mechanics. Computational Mechanics Advances (J. T. Ohen ed.), vol. 2. Lausanne: North-Holland, pp. 1-124.

Grisvard, P. (1985) Elliptic Problems in Nonsmooth Domains. Boston: Pitman Publishing.

HiptmaiR, R. (1999) Multigrid method for Maxwell's equations. SIAM J. Numer. Anal., 36, 204-225.

HU, Q. \& Zou, J. (2003) A non-overlapping domain decomposition method for Maxwell's equations in three dimensions. SIAM J. Numer. Anal., 41, 1682-1708.

HU, Q. \& ZOU, J. (2004) Substructuring preconditioners for saddle-point problems arising from Maxwell's equations in three dimensions. Math. Comput., 73, 35-61.

Klawonn, A., Rheinbach, O. \& Widlund, O. B. (2004) Some computational results for dual-primal FETI methods for three dimensional elliptic problems. Domain Decomposition Methods in Science and Engineering (R. Kornhuber, R. H. W. Hoppe, J. Périaux, O. Pironneau, O. B. Widlund \& J. Xu, eds). Lecture Notes in Computational Science and Engineering. Springer. (Proceedings of the 15th International Conference on Domain Decomposition Methods, Berlin, 21-25 July 2003.)

KLAWOnn, A. \& WidLund, O. B. (2004) Selecting constraints in dual-primal FETI methods for elasticity in three dimensions. Domain Decomposition Methods in Science and Engineering (R. Kornhuber, R. H. W. Hoppe, J. Périaux, O. Pironneau, O. B. Widlund \& J. Xu, eds). Lecture Notes in Computational Science and Engineering. Springer. (Proceedings of the 15th International Conference on Domain Decomposition Methods, Berlin, 21-25 July 2003.)

Klawonn, A., WidLund, O. B. \& DRYJA, M. (2002) Dual-primal FETI methods for three-dimensional elliptic problems with heterogeneous coefficients. SIAM J. Numer. Anal., 40, 159-179.

MANDEL, J. (1990) Two-level domain decomposition preconditioning for the p-version finite element version in three dimensions. Int. J. Numer. Meth. Eng., 29, 1095-1108. 
MAndel, J. \& BRezinA, M. (1996) Balancing domain decomposition for problems with large jumps in coefficients. Math. Comput., 65, 1387-1401.

MAndel, J. \& TeZAur, R. (2001) On the convergence of a dual-primal substructuring method. Numer. Math., 88, 543-558.

Monk, P. (2003) Finite element methods for Maxwell's equations. Numerical Mathematics and Scientific Computation. New York: Oxford University Press. Oxford Science Publications.

NÉDÉLEC, J.-C. (1980) Mixed finite elements in $R^{3}$. Numer. Math., 35, 315-341.

RAPETTI, F. \& TOSELLI, A. (2001) A FETI preconditioner for two dimensional edge element approximations of Maxwell's equations on non-matching grids. SIAM J. Sci. Comput., 23, 92-108.

Smith, B. F., Bjørstad, P. E. \& Gropp, W. D. (1996) Domain Decomposition: Parallel Multilevel Methods for Elliptic Partial Differential Equations. New York: Cambridge University Press.

Toselli, A. (2000a) Neumann-Neumann methods for vector field problems. Electron. Trans. Numer. Anal., 11, $1-24$.

Toselli, A. (2000b) Two iterative substructuring methods for Maxwell's equations with discontinuous coefficients in two dimensions. Twelfth International Symposium on Domain Decomposition Methods for Partial Differential Equations. (T. Chan, T. Kako, H. Kawarada \& O. Pironneau, eds). DDM.org.

Toselli, A. \& KLAWOnN, A. (2001) A FETI domain decomposition method for edge element approximations in two dimensions with discontinuous coefficients. SIAM J. Numer. Anal., 39, 932-956.

Toselli, A. \& VAsseur, X. (2004) Dual-primal FETI algorithms for edge element approximations: two-dimensional $h$ and $p$ finite elements on shape-regular meshes. Technical Report 04-01, Seminar for Applied Mathematics, ETH Zürich, 2004. SIAM J. Numer. Anal., 42, 2590-2611.

Toselli, A. \& Widlund, O. (2004) Domain Decomposition Methods: Theory and Algorithms. Springer Series in Computational Mathematics, vol. 34. New York: Springer.

Toselli, A., Widlund, O. B. \& Wohlmuth, B. I. (2001) An iterative substructuring method for Maxwell's equations in two dimensions. Math. Comput., 70, 935-949.

Traore, K. F., Farhat, C., Lesoinne, M. \& Dureisseix, D. (2002) A domain decomposition method with Lagrange multipliers for the massively parallel solution of large-scale contact problems.Proceedings of the Fifth World Congress on Computational Mechanics (WCCM V) (H. A. Mang, F. G. Rammerstorfer \& J. Eberhardsteiner, eds). Vienna University of Technology, Austria, 7-12 July 2002. http://wccm.tuwien.ac.uk

Wohlmuth, B., Toselli, A. \& Widlund, O. (2000) An iterative substructuring method for Nédélec finite elements in three dimensions (unpublished).

Wohlmuth, B. I., Toselli, A. \& Widlund, O. B. (2000) An iterative substructuring method for RaviartThomas vector fields in three dimensions. SIAM J. Numer. Anal., 37, 1657-1676.

\section{Appendix A. Proof of the Lemma 7.2}

We first consider the first inequality in Lemma 7.2. We need to show that, given a piecewise constant function $\zeta$ on $\partial \Omega_{i}$, we have

$$
\|\zeta\|_{H^{-1 / 2}(F)}^{2} \leqslant C(1+\log (H / h))^{2}\|\zeta\|_{H^{-1 / 2}\left(\partial \Omega_{i}\right)}^{2} .
$$

The proof relies on two results. The first is an inverse inequality for the Sobolev norms of negative exponent:

LemmA A.1 Let $\zeta$ be a piecewise constant function on $\partial \Omega_{i}$. Given $\epsilon \in[0,1 / 2]$, there exists a constant, independent of $\zeta, \epsilon, H_{i}$ and the mesh size, such that,

$$
\|\zeta\|_{H^{-1 / 2+\epsilon}\left(\partial \Omega_{i}\right)} \leqslant C h^{-\epsilon}\|\zeta\|_{H^{-1 / 2}\left(\partial \Omega_{i}\right)} .
$$


Proof. The proof relies on the inverse inequality

$$
\|\zeta\|_{L^{2}\left(\partial \Omega_{i}\right)} \leqslant C h^{-1 / 2}\|\zeta\|_{H^{-1 / 2}\left(\partial \Omega_{i}\right)}
$$

see Dahmen et al. (2004, Theorem 4.6), which gives a bound for the identity operator $I: H^{-1 / 2}\left(\partial \Omega_{i}\right) \rightarrow$ $L^{2}\left(\partial \Omega_{i}\right)$ restricted to the finite-element space on $\partial \Omega_{i}$, together with the trivial estimate for $I: H^{-1 / 2}$ $\left(\partial \Omega_{i}\right) \rightarrow H^{-1 / 2}\left(\partial \Omega_{i}\right)$, and an interpolation argument.

The second result is a well-known localization result. We give details about the proof since we need an explicit bound in $\epsilon$.

LemmA A.2 Let $\psi \in H^{1 / 2-\epsilon}(F)$, with $\epsilon \in(0,1 / 2)$, and $\mathcal{E} \psi$ its extension by zero to the whole of $\partial \Omega_{i}$. There exists a constant, independent of $\psi$ and $\epsilon$, such that

$$
\|\mathcal{E} \psi\|_{H^{1 / 2-\epsilon}\left(\partial \Omega_{i}\right)} \leqslant C \epsilon^{-1}\|\psi\|_{H^{1 / 2-\epsilon}(F)} .
$$

Proof. We first note that, thanks to Grisvard (1985, Lemma 1.3.2.6),

$$
\|\mathcal{E} \psi\|_{H^{1 / 2-\epsilon}\left(\partial \Omega_{i}\right)}^{2} \leqslant C\left(\|\psi\|_{H^{1 / 2-\epsilon}(F)}^{2}+\left\|\rho^{\epsilon-1 / 2} \psi\right\|_{L^{2}(F)}^{2}\right)
$$

with $\rho(x)$ the distance from a point $x$ to $\partial F$. Since, for the given $\epsilon$, the space $H^{1 / 2-\epsilon}(F)$ coincides with that of its extensions by zero to $\partial \Omega_{i}$, it is enough to show that

$$
\left\|\rho^{\epsilon-1 / 2} \psi\right\|_{L^{2}(F)} \leqslant C \epsilon^{-1}\|\psi\|_{H^{1 / 2-\epsilon}(F)} .
$$

This is indeed the statement of Grisvard (1985, Theorem 1.4.4.4), with $\Omega=F, p=2$ and $s=1 / 2-\epsilon$. The proof can be found there and it relies on the Hardy inequality

$$
\int_{0}^{+\infty}\left(\frac{1}{t} \int_{t}^{+\infty} \psi(s) \mathrm{d} s\right)^{2} t^{2 \alpha} \mathrm{d} t \leqslant(\alpha-1 / 2)^{-2} \int_{0}^{+\infty} \psi(t)^{2} t^{2 \alpha} \mathrm{d} t, \quad \alpha>\frac{1}{2}
$$

for which the explicit dependence of the constant has been derived from Grisvard (1985, p. 28).

We are now ready to prove (A.1). Lemma A.2 and a duality argument yield

$$
\|\zeta\|_{H^{-1 / 2+\epsilon}(F)} \leqslant C \epsilon^{-1}\|\zeta\|_{H^{-1 / 2+\epsilon}\left(\partial \Omega_{i}\right)}
$$

Using the inverse inequality of Lemma A.1, therefore, gives

$$
\|\zeta\|_{H^{-1 / 2}(F)} \leqslant C\|\zeta\|_{H^{-1 / 2+\epsilon}(F)} \leqslant C \epsilon^{-1}\|\zeta\|_{H^{-1 / 2+\epsilon}\left(\partial \Omega_{i}\right)} \leqslant C \epsilon^{-1} h^{-\epsilon}\|\zeta\|_{H^{-1 / 2}\left(\partial \Omega_{i}\right)}
$$

The proof is concluded by choosing $1 / \epsilon=1+\log (H / h)$.

As previously mentioned, a stronger version of (A.1) was already proven in Wohlmuth (2000, Lemma 4.4). The proof given here, however, has the advantage that only relies on the existence of the 
inverse inequality in Lemma A.1, which holds for more general finite-element spaces. The proof can be directly applied for the second inequality of Lemma 7.2, involving the subspaces of $\mathbf{H}^{-1 / 2}(F)$ and $\mathbf{H}_{\perp}^{-1 / 2}\left(\partial \Omega_{i}\right)$ consisting of tangential, edge-element vectors on $\partial \Omega_{i}$. More precisely, given a tangential vector $\mathbf{w}$ on $\partial \Omega_{i}$, we define

$$
\|\mathbf{w}\|_{\mathbf{H}_{\perp}^{1 / 2}\left(\partial \Omega_{i}\right)}^{2}=\sum_{F^{\prime}}\|\mathbf{w}\|_{\mathbf{H}^{1 / 2}\left(F^{\prime}\right)}^{2}+\sum_{F^{\prime}} \sum_{F^{\prime \prime} \neq F^{\prime}} \int_{F^{\prime}} \int_{F^{\prime \prime}} \frac{\left|\mathbf{w}\left(x^{\prime}\right) \cdot \boldsymbol{v}^{\prime}-\mathbf{w}\left(x^{\prime \prime}\right) \cdot \boldsymbol{v}^{\prime \prime}\right|^{2}}{\left|x^{\prime}-x^{\prime \prime}\right|^{3}} \mathrm{~d} S^{\prime} \mathrm{d} S^{\prime \prime} ;
$$

see Buffa \& Ciarlet (2001a), where the last sum is taken over the faces $F^{\prime \prime}$ that share an edge with $F^{\prime}$. Here, given two faces that share an edge, we have employed the vectors

$$
\boldsymbol{v}^{\prime}=\mathbf{t}_{\partial F^{\prime}} \times \mathbf{n}^{\prime}, \quad \boldsymbol{v}^{\prime \prime}=\mathbf{t}_{\partial F^{\prime \prime}} \times \mathbf{n}^{\prime \prime},
$$

with the outward unit normal vectors $\mathbf{n}^{\prime}$ and $\mathbf{n}^{\prime \prime}$ to the two faces, and the assumption that $\mathbf{t}_{\partial F^{\prime}}$ and $\mathbf{t}_{\partial F^{\prime \prime}}$ have the same direction along the common edge. We note that the last term in (A.2) scales like the $H^{1 / 2}$ seminorm and therefore no scaling factor involving the diameter $H_{i}$ needs to be employed; see

the definition of scaled norms in Section 7.1. Given this norm, we can define a norm in $\mathbf{H}_{\perp}^{-1 / 2}\left(\partial \Omega_{i}\right)$ by duality. Lemmas A.1 and A.2 remain valid in this case. Finally, the third inequality of Lemma 7.2 is proven by a scaling argument.

\section{Appendix B. Proof of Lemma 7.12}

For this section we employ the lowest-order Raviart-Thomas space $R T^{h}\left(\Omega_{i}\right)$, conforming in $H\left(\operatorname{div} ; \Omega_{i}\right)$, and need to recall a few results. We refer to Brezzi \& Fortin (1991, Section III.3.2) or Nédélec (1980), Amrouche et al. (1998) and Hiptmair (1999) for an introduction. Here, $H$ (div; $\Omega_{i}$ ) is the space of square-summable vectors in $\Omega_{i}$ with square-summable divergence and $H_{\star}\left(\operatorname{div} ; \Omega_{i}\right)$ its subspace of vectors with vanishing normal component on $\partial \Omega_{i} \cap \partial \Omega$. For the reference cube $K=\hat{Q}$, we define

$$
R T(K)=\mathbb{Q}_{1,0,0}(K) \otimes \mathbb{Q}_{0,1,0}(K) \otimes \mathbb{Q}_{0,0,1}(K) ;
$$

for an affinely mapped element, the definition can be found in Brezzi \& Fortin (1991, Section III.3.2). We then set

$$
R T^{h}\left(\Omega_{i}\right):=\left\{\mathbf{u} \in \mathrm{H}_{\star}\left(\operatorname{div} ; \Omega_{i}\right) \mid \mathbf{u}_{\left.\right|_{K}} \in R T(K), K \in \mathcal{T}_{h}, K \subset \Omega_{i}\right\} .
$$

We recall that for conforming finite elements in $H\left(\operatorname{div} ; \Omega_{i}\right)$ the normal component across element boundaries is continuous. In addition, the normal component $\mathbf{u} \cdot \mathbf{n}$ of a vector $\mathbf{u} \in R T^{h}\left(\Omega_{i}\right)$ on $\partial \Omega_{i}$ is a piecewise constant function.

We next define $N D_{F}^{h}\left(\Omega_{i}\right)$ and $V_{F}^{h}\left(\Omega_{i}\right)$ as the subspaces of $N D^{h}\left(\Omega_{i}\right)$ and $V^{h}\left(\Omega_{i}\right)$, respectively, of tangential vectors and functions that vanish on $\partial \Omega_{i} \backslash F$. Analogously, we define $R T_{F}^{h}\left(\Omega_{i}\right)$ as the subspace of $R T^{h}\left(\Omega_{i}\right)$ of vectors with vanishing normal component outside $F$. We summarize some of the properties that we need in the following lemma.

LEMMA B. 1

1. We have the inclusion $\nabla V_{F}^{h}\left(\Omega_{i}\right) \subset N D_{F}^{h}\left(\Omega_{i}\right)$. The following orthogonal decomposition is therefore well defined

$$
N D_{F}^{h}\left(\Omega_{i}\right)=\nabla V_{F}^{h}\left(\Omega_{i}\right) \oplus N D_{F}^{h}\left(\Omega_{i}\right)^{\perp} .
$$


2. We have curl $N D_{F}^{h}\left(\Omega_{i}\right) \subset R T_{F}^{h}\left(\Omega_{i}\right)$ and, for every $\mathbf{v} \in \operatorname{curl} N D_{F}^{h}\left(\Omega_{i}\right)$ there is a unique $\mathbf{u}^{\perp} \in$ $N D_{F}^{h}\left(\Omega_{i}\right)^{\perp}$, such that

$$
\operatorname{curl} \mathbf{u}^{\perp}=\mathbf{v}, \quad\left\|\mathbf{u}^{\perp}\right\|_{L^{2}\left(\Omega_{i}\right)}^{2} \leqslant C H_{i}^{2}\left\|\operatorname{curl} \mathbf{u}^{\perp}\right\|_{L^{2}\left(\Omega_{i}\right)}^{2}
$$

Conversely, if $\mathbf{v} \in R T_{F}^{h}\left(\Omega_{i}\right)$ has a vanishing divergence, there exists $\mathbf{u} \in N D_{F}^{h}\left(\Omega_{i}\right)$, such that $\mathbf{v}=$ curl $\mathbf{u}$.

3. Let $\mathbf{u}$ be the restriction of a tangential vector in $W_{i}$ to the face $F$, such that $\mathbf{u} \cdot \mathbf{t}_{\partial F}=0$ and $\operatorname{curl}_{S} \mathbf{u}=0$. Then, there exists a continuous piecewise bilinear function $\phi$ on $F$ that vanishes on $\partial F$, such that $\mathbf{u}=\nabla_{S} \phi$.

For the proof, the results for the spaces $N D^{h}\left(\Omega_{i}\right)$ and $N D_{0}^{h}\left(\Omega_{i}\right)$ in Amrouche et al. (1998, Section 4.1) can be straightforwardly generalized to $N D_{F}^{h}\left(\Omega_{i}\right)$. We note that we have excluded the case when a face coincides with a connected component of $\partial \Omega_{i}$; see Section 3. For (B.2) we refer, in particular, to Amrouche et al. (1998, Proposition 4.6), where, as usual, the dependence on $H_{i}$ is obtained by a scaling argument. Point 3 is a 2D result for a face $F$ and can be proven as in Amrouche et al. (1998, Lemma 4.3).

We next need an extension theorem, which was originally given in Wohlmuth (2000, Lemma 4.3).

LEMMA B. 2 Let $\zeta$ be a piecewise constant function on $\partial \Omega_{i}$ with vanishing mean value. Then there exists an extension $\mathbf{v}(\zeta) \in R T^{h}\left(\Omega_{i}\right)$, such that $\operatorname{div} \mathbf{v}(\zeta)=0$ and

$$
\begin{aligned}
& \mathbf{v}(\zeta) \cdot \mathbf{n}=\zeta, \quad \text { on } \partial \Omega_{i}, \\
& \|\mathbf{v}(\zeta)\|_{L^{2}\left(\Omega_{i}\right)} \leqslant C\|\zeta\|_{H^{-1 / 2}\left(\partial \Omega_{i}\right)},
\end{aligned}
$$

with a constant that is independent of the mesh size and $H_{i}$.

We finally need a result for continuous functions.

LEMMA B.3 Let $\phi$ be a continuous, piecewise bilinear function on $F$ that vanishes on $\partial F$ and $\mathcal{E}$ the operator that defines the extension by zero to $\partial \Omega_{i}$. Then,

$$
\|\phi\|_{H_{00}^{1 / 2}(F)}=\|\mathcal{E} \phi\|_{H^{1 / 2}\left(\partial \Omega_{i}\right)} \leqslant C\left\|\mathcal{E}\left(\nabla_{S} \phi\right)\right\|_{\mathbf{H}_{\perp}^{-1 / 2}\left(\partial \Omega_{i}\right)} .
$$

Proof. We first recall that the surface divergence operator on $\partial \Omega_{i}$

$$
\operatorname{div}_{S}: \mathbf{H}_{\perp}^{1 / 2}\left(\partial \Omega_{i}\right) \longrightarrow H^{-1 / 2}\left(\partial \Omega_{i}\right)
$$

is defined as the adjoint of $\nabla_{S}$ and is continuous and surjective; see Buffa \& Ciarlet (2001b), Proposition 4.7 and Reminder 4.8. We can therefore write

$$
\|\mathcal{E} \phi\|_{H^{1 / 2}\left(\partial \Omega_{i}\right)}=\sup _{\psi \in H^{-1 / 2}\left(\partial \Omega_{i}\right)} \frac{\langle\psi, \mathcal{E} \phi\rangle}{\|\psi\|_{H^{-1 / 2}\left(\partial \Omega_{i}\right)}} \leqslant C \sup _{\mathbf{v} \in \mathbf{H}_{\perp}^{1 / 2}\left(\partial \Omega_{i}\right)} \frac{\left\langle\operatorname{div}_{S} \mathbf{v}, \mathcal{E} \phi\right\rangle}{\|\mathbf{v}\|_{\mathbf{H}_{\perp}^{1 / 2}\left(\partial \Omega_{i}\right)}},
$$

with $\langle\cdot, \cdot\rangle$ the obvious duality pairing. Using the definition of $\operatorname{div}_{S}$ as adjoint of $\nabla_{S}$ and the fact that

$$
\nabla_{S}(\mathcal{E} \phi)=\mathcal{E}\left(\nabla_{S} \phi\right)
$$

completes the proof. 
We are now ready to prove Lemma 7.12. Some of the ideas of the proof rely on Wohlmuth (2000). We first consider the curl of the tangential vector $\tilde{\mathbf{w}}_{F}$ :

$$
\zeta_{F}=\operatorname{curl}_{S} \tilde{\mathbf{w}}_{F}
$$

and note that, since $\tilde{\mathbf{w}}_{F}$ has a vanishing tangential component along $\partial F, \zeta_{F}$ has mean value zero on $F$. We then consider the extension by zero $\zeta=\mathcal{E}\left(\zeta_{F}\right)$ to $\partial \Omega_{i}$. This extension satisfies the assumptions of Lemma B.2. We can therefore define the divergence-free extension

$$
\mathbf{v}_{F}=\mathbf{v}(\zeta)
$$

in $\Omega_{i}$. Since $\mathbf{v}_{F} \in R T_{F}^{h}\left(\Omega_{i}\right)$ is divergence free, Lemma B.1, point 2, ensures that there exists a vector $\mathbf{u}_{F} \in N D_{F}^{h}\left(\Omega_{i}\right)^{\perp}$, such that

$$
\operatorname{curl} \mathbf{u}_{F}=\mathbf{v}_{F} .
$$

Lemma B.2 and (7.2) thus provide the first of (7.9):

$$
\left\|\operatorname{curl} \mathbf{u}_{F}\right\|_{L^{2}\left(\Omega_{i}\right)} \leqslant C\|\zeta\|_{H^{-1 / 2}\left(\partial \Omega_{i}\right)} \leqslant C\left\|\zeta_{F}\right\|_{H^{-1 / 2}(F)}=C\left\|\operatorname{curl}_{S} \mathbf{u}_{F}\right\|_{H^{-1 / 2}(F)} .
$$

The second inequality of (7.9) is a consequence of (B.2).

We next restrict $\mathbf{u}_{F}$ to $F$. Since

$$
\operatorname{curl}_{S}\left(\tilde{\mathbf{w}}_{F}-\mathbf{u}_{F}\right)=0,
$$

Lemma B.1, point 3, ensures that there exists a continuous, piecewise bilinear function that vanishes on $\partial F$, such that (7.8) holds. We then extend $\phi_{F}$ by zero to the rest of $\partial \Omega_{i}$ and consider its extension to $\Omega_{i}$ which is the Laplace discrete harmonic. A standard stability result for Laplace discrete harmonic functions and Lemma B.3 yields

$$
\begin{aligned}
\left\|\nabla \phi_{F}\right\|_{L^{2}\left(\Omega_{i}\right)} & \leqslant C\left|\phi_{F}\right|_{H_{00}^{1 / 2}(F)} \leqslant C\left\|\mathcal{E}\left(\nabla_{S} \phi_{F}\right)\right\|_{\mathbf{H}_{\perp}^{-1 / 2}\left(\partial \Omega_{i}\right)} \\
& \leqslant C\left\|\mathcal{E} \tilde{\mathbf{w}}_{F}\right\|_{\mathbf{H}_{\perp}^{-1 / 2}\left(\partial \Omega_{i}\right)}+\left\|\mathcal{E} \mathbf{u}_{F}\right\|_{\mathbf{H}_{\perp}^{-1 / 2}\left(\partial \Omega_{i}\right)} \\
& \leqslant C\left\|\tilde{\mathbf{w}}_{F}\right\|_{\mathbf{H}^{-1 / 2}(F)}+\left\|\mathcal{E} \mathbf{u}_{F}\right\|_{\mathbf{H}_{\perp}^{-1 / 2}\left(\partial \Omega_{i}\right)}
\end{aligned}
$$

where we have used (7.2) for the last inequality. We are only left with the task of finding a bound for the term in $\mathbf{u}_{F}$. For this, we can use the trace estimate in Lemma 7.1, the definition of $\mathbf{u}_{F}$ and the two already proven bounds:

$$
\begin{aligned}
\left\|\mathcal{E} \mathbf{u}_{F}\right\|_{\mathbf{H}_{\perp}^{-1 / 2}\left(\partial \Omega_{i}\right)} & =\left\|\mathbf{u}_{F}\right\|_{\mathbf{H}_{\perp}^{-1 / 2}\left(\partial \Omega_{i}\right)} \leqslant C\left(\left\|\mathbf{u}_{F}\right\|_{L^{2}\left(\Omega_{i}\right)}^{2}+H_{i}^{2}\left\|\operatorname{curl} \mathbf{u}_{F}\right\|_{L^{2}\left(\Omega_{i}\right)}^{2}\right) \\
& \leqslant C H_{i}^{2}\left\|\operatorname{curl} \mathbf{u}_{F}\right\|_{L^{2}\left(\Omega_{i}\right)}^{2} \leqslant C H_{i}^{2}\left\|\operatorname{curl}_{S} \mathbf{u}_{F}\right\|_{H^{-1 / 2}(F)}^{2} .
\end{aligned}
$$

